\title{
Vehicle and mission design of a future small payload launcher
}

\author{
Christie Alisa Maddock, Federico Toso ${ }^{\dagger}$ Lorenzo Ricciardi†, Alessandro Mogavero ${ }^{\dagger}$ \\ University of Strathclyde, Glasgow, G1 1XJ, United Kingdom \\ Kin Hing Lo ${ }^{\dagger}$ Sriram Rengarajan† Konstantinos Kontis ${ }^{\ddagger}$ \\ University of Glasgow, G12 8QQ, United Kingdom \\ Andy Milne§, Jim Merrifield, and David Evans" \\ Fluid Gravity Engineering, Hants, PO10 7DX, United Kingdom \\ Michael West** \\ BAE Systems Regional Aircraft, Prestwick, KA9 2RW, United Kingdom \\ Stuart McIntyre ${ }^{\dagger \dagger}$ \\ Orbital Access Limited, Prestwick, KA9 2RW, United Kingdom
}

\begin{abstract}
This paper presents the conceptual design and performance analysis of a partially reusable space launch vehicle for small payloads. The system uses a multi-stage vehicle with rocket engines, with a reusable first stage capable of glided or powered flight, and expendable upper stage(s) to inject a $500 \mathrm{~kg}$ payload in different low Earth orbits. The space access vehicle is designed to be air-launched from a modified aircraft carrier. The aim of the system design is to develop a commercially viable launch system for near-term operation, thus emphasis is placed on the efficient use of high TRL technologies. The vehicle design are analysed using a multi-disciplinary design optimisation approach to evaluate the performance, operational capabilities and design trade-offs.
\end{abstract}

\section{Introduction}

ANY of the forecast studies looking at the future of the satellite market predict a period of unprecedented

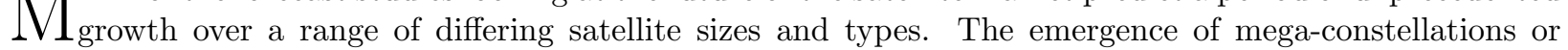
smaller single purpose satellite constellations exploit the ever progressing miniaturisation of powerful sensors capable of capturing data or providing services that were hitherto only feasibly carried on large government agency developed satellites. Combined with the inexorable development of high powered computing and high bandwidth communications, small satellites can now deliver space based applications with levels of fidelity and performance never previously accessible by broad based commercial users. The investment by many countries, including the UK, in satellite application development and entrepreneurial growth in space based service development is a major contributing factor into the forecasted growth in small satellite demand.

With the increased demand, there is increased pressure for new accessible, responsive and cost effective small satellite launch capacity. Yet the austere period following the termination of the Shuttle programme has not yielded many proven small satellite launch technologies. The focus for many space agencies, in

\footnotetext{
*Lecturer of Aerospace Engineering, christie.maddock@strath.ac.uk, AIAA Member

${ }^{\dagger}$ Research Associate, AIAA Member/Student Member

${ }^{\ddagger}$ Mechan Chair of Engineering, Professor of Aerospace Engineering, AIAA Fellow

$\S$ Senior Scientist

ๆ Director, Civil Space

" Project Manager

** Chief Aerodynamicist and Head of Technology

${ }^{\dagger \dagger}$ Chief Executive Officer
} 
particular NASA, on enabling human spaceflight through programmes such as the X Prize in the early 2000s meant that entrepreneurial investment has mainly been focused on human spaceflight capabilities and in replacing the ISS resupply capacity lost with the termination of the Shuttle programme. With the recent shift towards fully commercial options for space launch, coupled with the current and predicted growth of the market, there has been a resurgence to develop and operate a small payload launch system. A survey from $2015^{1}$ lists 20 launch vehicles under development around the world designed to launch small satellite payloads weighing up to $1000 \mathrm{~kg}$. Most are in development by small to medium sized businesses, with a target first flight by 2020 .

The following paper details the conceptual design and analysis of a future commercial launch system for small payloads (up to $500 \mathrm{~kg}$ ). The system is a multi-stage vehicle using rocket propulsion systems that will be air-launched from a carrier aircraft. The main vehicle is a spaceplane design that will allow for glided re-entry/return flight. The second stage is stored within the main body of the spaceplane, among other benefits this allows for better control of the moments induced by the movement of the centre of gravity though introduces complexity and release issues. An optional small upper stage is also investigated to increase the range of possible orbits that can be reached. With the main operational spaceport located on the western coast of Scotland, the air-launch increases the type of orbits that can be reached, and improves the flexibility of the system by allowing the transport and recovery of the first stage.

The paper describes the overall approach with design objectives and mission requirements, then details the system models developed for use within a specialised integrated design platform for space access vehicles. The optimisation used within the system performance analysis is described, with results presented examining the trade-off in performance of altering key design variables in the configuration, specifically the engine and wing sizing (aerodynamic efficiency). The nominal mission is to deploy a $500 \mathrm{~kg}$ payload into $600 \mathrm{~km}$ altitude circular orbit at an inclination of $88.2 \mathrm{deg}$, with an option for an upper stage to raise the orbital altitude to deliver a $150 \mathrm{~kg}$ payload to $1200 \mathrm{~km}$.

\section{Approach}

A specialised integrated design platform was used that was developed to analyse the performance and optimise the mission design for transatmospheric flight vehicles. The software has been used to evaluate different space launch systems, ${ }^{2,3}$ from single stage to orbit vehicles ${ }^{4}$ to expendable vertically launched rockets. ${ }^{5}$ Computationally fast engineering models were developed for the different subsystems to allow the performance of the system to be evaluated using a multi-disciplinary design optimisation approach. Different design criteria were selected as inputs, with the models relating the impact of changes on those variables on the system. For example, the wing reference area is a design input that affects the aerodynamic lift and drag forces, and the vehicle dry mass. A full mission is simulated, optimising different criteria with various system and operational constraints. Target orbits and payloads were determined through a market demand study. ${ }^{6,7}$

\section{System models}

In this section, mathematical models are presented for the vehicle design and operation. The models are divided by discipline: vehicle mass and configuration, aerodynamics, aerothermodynamics, propulsion, environment models for Earth including geometry, gravitational field and atmospheric model, and the flight dynamics and control.

\section{III.A. Vehicle configuration}

The basic concept for the launch system was for operations from horizontal take-off spaceports. Early in the concept analysis it was decided that for maximum operational flexibility the concept of an air launched system would be investigated. This drove the basic configuration which consisted of a carrier aircraft, converted from a large commercial airliner, a winged recoverable booster and an expendable upper stage. Two launch vehicle configuration concepts were considered: a more conventional, horizontal stack configuration where the upper stage and payload are attached to the front of the winged booster stage, and a larger winged booster with the upper stage and payload housed in an internal payload bay.

Through the course of the study the concept of the winged recoverable booster evolved from a rocket 
with wings and a payload bay, to an integrated aerospace plane with multiple parallel propellant tanks and a novel payload cartridge system (see Fig. 1).

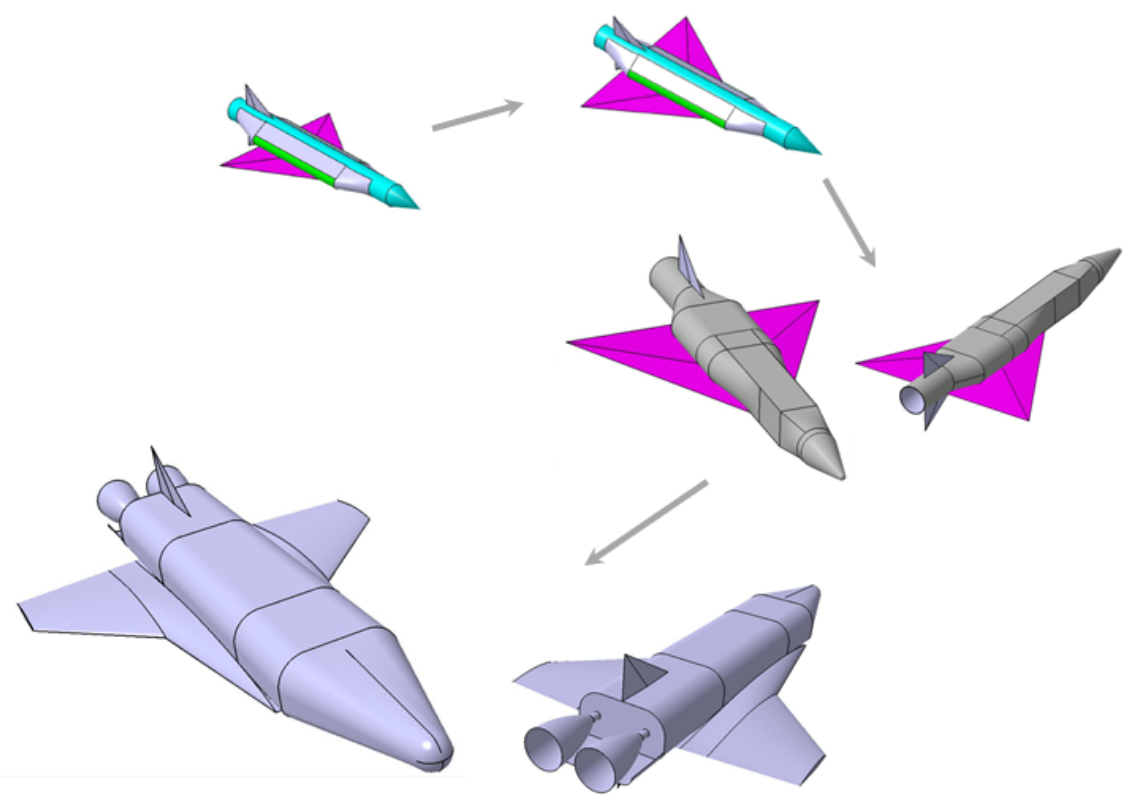

Figure 1. Conceptual drawings showing the evolution of the configuration

For the concept studied, the launch vehicle was mounted below the carrier aircraft on the fuselage centreline. This layout was chosen primarily for flight safety reasons, as release of the launch vehicle in a power off glide is most readily achieved by a conventional drop manoeuvre, in a similar fashion to the Pegasus/L-1011 combination operated by Orbital ATK. This drove space envelope and mass constraints on the launch vehicle.

The initial vehicle layout employed a $60^{\circ}$ sweep delta wing. Initial sizing was based on wing loading requirements for manoeuvre capability at maximum mass after release and for good low speed handling at the empty mass for approach and landing. Previous data for winged orbital re-entry vehicles, research aircraft, air dropped vehicles, high performance military aircraft and high performance civil aircraft were used to inform the wing sizing and concept layout models were produced in CATIA. These models were subsequently used for the engineering analysis work.

During the study the mission requirements evolved and the vehicle concept was developed into a more complex configuration to meet these requirements. The final configuration had a straight tapered wing of $45^{\circ}$ leading edge sweep featuring an $80^{\circ}$ sweep inboard leading edge extension. To accommodate the payload cartridge system the fuselage width was approximately doubled with respect to the original concept. This allowed the propellant tanks to be distributed in a manner conducive to good control of the centre of gravity during the powered ascent phase of flight. It also gave a lifting fuselage shape.

A number of parametric mass prediction methods were used for the initial concept level mass predictions, alongside mass data from a NASA reusable launch vehicle study. Methods were sourced from a methods database document produced by Rohrschneider. ${ }^{8}$ The data were applied carefully based on the quoted sources. A comparison was made with a NASA study ${ }^{9}$ which helped to inform certain aspects of the final mass statement.

Full mass statements were prepared breaking the vehicle down into its major structural components (e.g., wing, fins, fuselage structure, propellant tanks) and the major systems (e.g., propulsion, avionics, landing gear). To allow for resizing during the vehicle optimisation phase, parametric scaling equations of the form,

$$
m_{\text {new }}=m_{\text {ref }}\left(\frac{S_{\text {new }}}{S_{\text {ref }}}\right)^{b}
$$

were developed for the major components, where $m$ is the mass, $S$ is a reference value which is scaled, $b$ a scaling exponent, and the subscripts ref refer to the original value and new to the scaled value. 
Knowing the mass breakdown and component layouts, the vehicle centre of gravity and its variation with fuel burn and payload deployment was determined and assessments made of the ability to trim i.e., reduce the pitching moment to zero during ascent and re-entry. Following this the propellant tanks were redistributed to give an acceptable centre of gravity range during flight. The internal layout of the final configuration is shown in Figure 2. Note that the propulsion system shown is indicative of the size and location but does not include any engineering details of the installation.

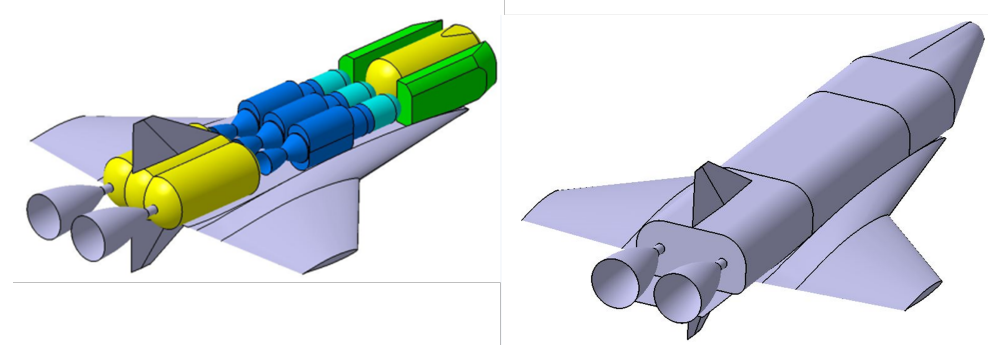

Figure 2. Final vehicle configuration including a possible internal layout

\section{III.B. Aerodynamics}

The aerodynamics of the vehicle configuration were analysed for Mach numbers ranging from 0.2 to 30 , angles of attack of $-5^{\circ}$ to $40^{\circ}$ and for altitudes up to $100 \mathrm{~km}$. The approach estimates the drag coefficient at zero incidence $C_{D_{0}}$ and the normal force coefficient $C_{N}$ at different angles of attack $\alpha$ for each component of the vehicle (fuselage, fairing, wings and tail). The coefficients are determined based on different theories for each Mach number range, from subsonic to hypersonic, detailed by Mason ${ }^{10}$ and Fleeman. ${ }^{11}$

The lift and drag forces of each component at different Mach numbers and angles of attack are modelled by,

$$
\begin{aligned}
& C_{L}=C_{N} \cos \alpha-C_{D_{0}} \sin \alpha \\
& C_{D}=C_{N} \sin \alpha+C_{D_{0}} \cos \alpha
\end{aligned}
$$

Equation 2 is valid for small angles of attack, when the axial force is approximately equal to drag. Although large angles of attack are considered the method would overpredict the lift at such angles, since the effect of stall is not accounted for. However, the effect of flow separation at the base of the fuselage is considered. The fuselage of the vehicle is approximated to have an elliptic cross section (with same area of cross section and major axis equal to half of the maximum width of the fuselage) in order to enable the application of theories. The lift and drag coefficients, after appropriate normalization (using the wing surface area) are then added up to give the total lift and drag coefficient of the entire configuration. Application of linear theory and modified Newtonion theory is used to deduce the wave drag coefficient at zero incidence over slender circular/elliptic nose $C_{d 0, w a v e, b}$, wave drag coefficient at zero incidence over the delta wing (as well as tail, which has similar form) $C_{d 0, \text { wave,w}}$, and the normal force coefficient as a function of angle of attack for the cone-cylinder $C_{N, b}$ as well as wings $C_{N, w}$, given by the following equations.

$$
\begin{array}{cr}
C_{d 0, \text { wave }, b}=0 & \text { for } M<1 \\
C_{d 0, \text { wave }, b}=\frac{3.6 d_{N}}{\ell_{N}(M-1)+3} & \text { for } M \geq 1 \\
C_{d 0, \text { wave }, w}=0 & \text { for } M<1 \\
C_{d 0, \text { wave }, w}=f\left(M_{\lambda L E}, \gamma, \delta_{L E}, t b / S_{w}\right) & \text { for } M \geq 1 \\
\left|C_{N, b}\right|=\frac{a_{N}}{b_{N}} \sin (2 \alpha) \cos (\alpha / 2)+2 \frac{\ell_{C}}{d_{C}} &
\end{array}
$$




$$
\begin{array}{ll}
\left|C_{N, w}\right|=\frac{\pi A}{2}|\sin \alpha \cos \alpha|+2 \sin ^{2} \alpha & \text { for } M^{2}<1+(8 / \pi A)^{2} \\
\left|C_{N, w}\right|=\frac{4|\sin \alpha \cos \alpha|}{\left(M^{2}-1\right)^{1 / 2}}+2 \sin ^{2} \alpha & \text { for } M^{2} \geq 1+(8 / \pi A)^{2}
\end{array}
$$

where $\ell_{N}$ is the length of the cone nose, $d_{N}$ is the equivalent diameter with major axis $a_{N}$ and minor axis $b_{N}, \ell_{C}$ is the length of the cylindrical body, $A$ is the aspect ratio of the wing, $t$ is the wing thickness, $b$ is the wing width, $S_{w}$ wing reference area, $\delta_{L E}$ is the wing thickness angle, $\gamma$ is the specific heat ratio, $\alpha$ is the angle of attack, and $M$ is the freestream Mach number with $M_{\lambda L E}$ the Mach number resolved in the direction normal to the wing leading edge with a sweep angle $\lambda_{L E}$.

The base wave drag on the wing $C_{d 0, w a v e, w}$ has a complex algebraic functional form $f$ whose expansion is given by Fleeman. ${ }^{11}$ The above coefficients are all normalized by their respective reference areas (and not a common reference area).

The coast drag of the cone-cylinder body $C_{d 0, c}$ is given by the following engineering correlation. ${ }^{11}$

$$
\begin{array}{ll}
C_{d 0, c}=0.12+0.13 M^{2} & \text { for } M<1 \\
C_{d 0, c}=0.25 / M & \text { for } M \geq 1
\end{array}
$$

The inviscid drag at zero incidence also includes drag due to nose and leading edge bluntness, which are also estimated using the semi-empirical expressions given by Fleeman. ${ }^{11}$

Severe peak heat transfer rates are among the important issues at hypersonic flow conditions. Blunt noses and leading edges are particularly preferred at hypersonic speeds in order to reduce the peak heat transfer rate. This would however increase the drag. A configuration that was initially considered for the study consisted of sharp nose and leading edges. A preliminary analysis was done at different hypersonic Mach numbers, introducing various bluntness radii at the nose and leading edge, predicting the peak heating theoretically ${ }^{12}$ at hypersonic Mach numbers, and the corresponding increase in drag due to bluntness. At Mach 8, a bluntness radius of $180 \mathrm{~mm}$ (20\% of the fuselage radius of the initial configuration, almost comparable with the equivalent radius of the present configuration) is found to significantly bring down the peak heating, by an order of magnitude, while the increase in the nose wave drag is within $10 \%$. A wing leading edge with a radius of $16 \mathrm{~mm}$ resulted in substantial decrease in stagnation heating, while increasing the leading edge wave drag by $15 \%$. In addition the introduction of nose bluntness also has the advantage of pushing the nose shock away from the wing tips; the bow shock profile was computed using semi-empirical correlations ${ }^{13}$ in order to monitor its position relative to the wing. Clearly the inclusion of bluntness is inevitable in order to manage the issues encountered in hypersonic flows. Accordingly, the present vehicle configuration consists of nose and leading edges with significant bluntness radii, and the drag due to the bluntness are a part of the total drag. A more detailed aerothermodynamic analysis is presented in Section III.C.

The inviscid coefficients are only dependent on Mach number and angle of attack and independent of altitude (independence with Reynolds number). However, the contribution of skin friction which is dependent of Reynolds number, leads to altitude dependence of the force coefficients. The skin friction drag coefficient at zero incidence for the cone-cylinder body $C_{D 0, f, b}$ and for the wing $C_{D 0, f, w}$ (tail too has similar functional form) are given by the following engineering correlations.

$$
\begin{aligned}
C_{d 0, f, b} & =0.053 \frac{\ell}{d}\left(\frac{M}{q \ell}\right)^{0.2} \\
C_{d 0, f, w} & =\frac{0.0266}{\left(q c_{\max }\right)^{0.2}}
\end{aligned}
$$

In the above equations $q$ is the dynamic pressure and $c_{\max }$ is the length of mean wing chord. The skin friction drag coefficient is added to the inviscid drag coefficients at zero incidence (for each component). The total drag coefficient at zero incidence together with the normal force coefficients are then used to calculate the lift and drag coefficients due to each component using Equation 2. The explicit density and velocity dependence of dynamic pressure leads to the altitude (and Reynolds number) dependence of the skin friction drag coefficient implicitly. The altitude dependence of the drag at zero dependence is exemplified in Fig. 3 for the initial configuration. There is an obvious and significant increase in drag, particularly at subsonic 


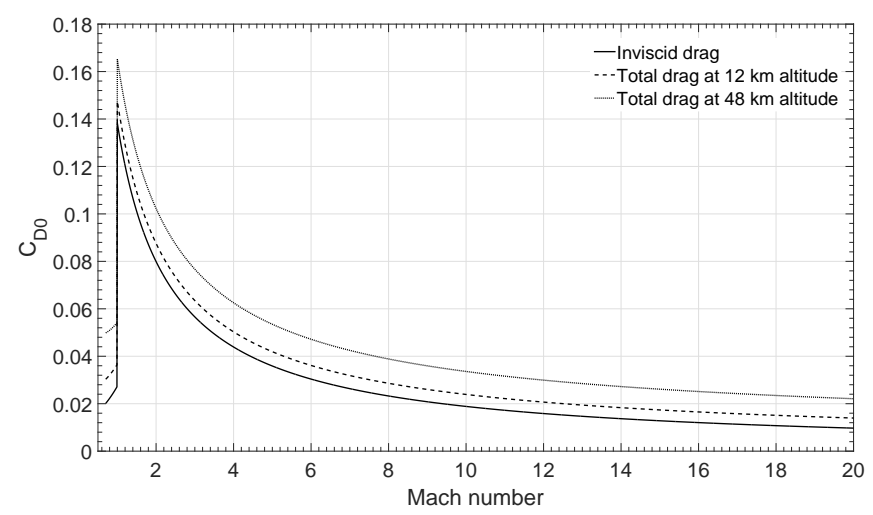

Figure 3. Altitude dependence of drag coefficient at zero incidence

and very high Mach numbers. Further, it can be noted that the total drag at $48 \mathrm{~km}$ altitude is much higher than that at $12 \mathrm{~km}$ altitude. The lower density contributes to the increased skin friction drag coefficient.

The method is validated using wind tunnel data at Mach 2,3 and $4^{14}$ and using gun tunnel data at Mach 8.2 for a simple cone-cylinder configuration as well as a cone-cylinder with a pair of delta wings using gun-tunnel data. ${ }^{15}$ The drag predictions at zero incidence for cone-cylinder match well with experiments. A comparison of the lift and drag coefficients predicted using the method with the Mach 8.2 gun tunnel data for the configuration of cone-cylinder with wings is shown in Fig. 4. It can be seen that the addition of skin friction does not greatly alter the predicted lift coefficient; the comparison with experiments is good for an angle of $8^{\circ}$, after which the method starts to over-predict the lift. The drag is significantly under-predicted by the inviscid model; however, with the addition of skin friction, the overall drag is slightly over-predicted. This trend is observed in other Mach numbers too; in general, for the cone-cylinder with wings it is found that the method starts over-predicting lift from angle of attack of around $10^{\circ}$, sometime by over $35 \%$. This is because the wing stall is not presently considered. The drag for the wing configuration is also generally over-predicted, therefore giving a conservative estimate.
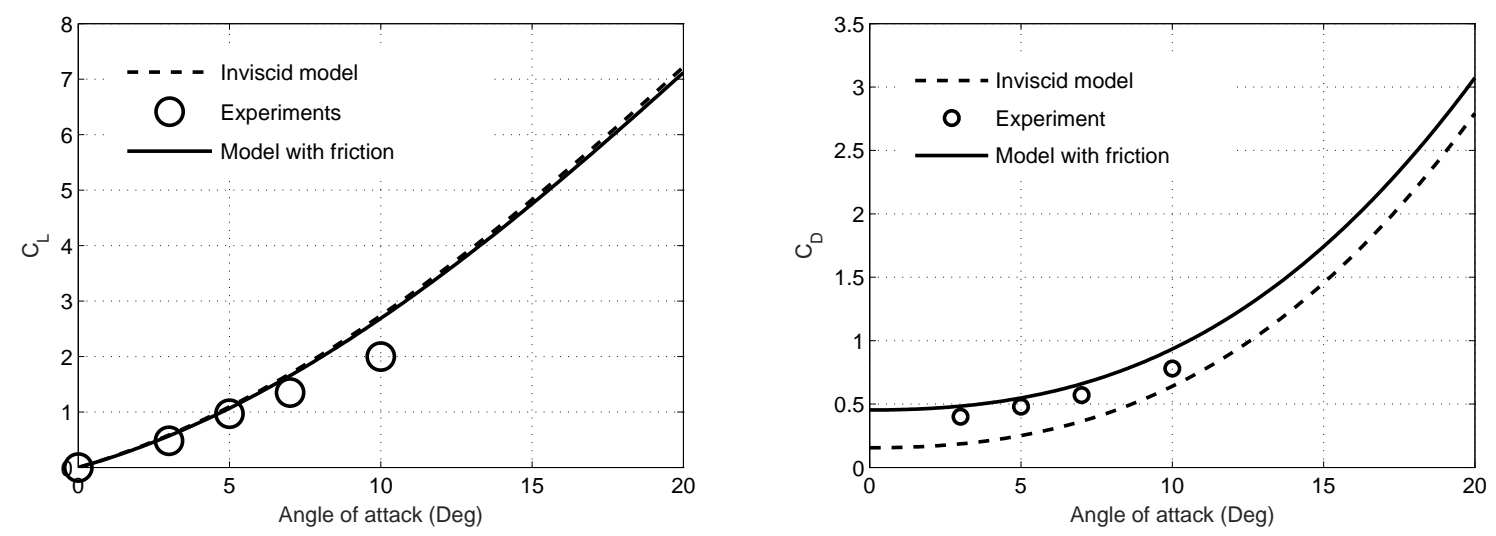

Figure 4. Validation of the models at Mach $8.2^{15}$

The inviscid lift and drag coefficients at different angles (in radians) and Mach numbers predicted are shown in Fig. 5. With increasing angle of attack, both the lift and drag coefficients increase. There is a sharp discontinuity in both the coefficients at transonic speed. After Mach 1, the lift coefficient increases and the drag coefficient decreases asymptotically with Mach number for any given (positive) angle of attack. With increasing angle both the lift and drag increases. High values of lift to drag ratio at subsonic speed is because of neglecting the skin friction which is important at low speeds especially for wings. Inclusion of skin friction is found to greatly alter the drag, especially at high altitudes as explained before. Thus with the addition of the contribution of skin friction, the lift and drag coefficients for each individual components as well as for the whole vehicle configuration becomes a function of Mach number, angle of attack and altitude; 
thus the aerodynamic data of force coefficients is generated as three-dimensional arrays which, along with the aero-thermal models, is used in the subsequent analysis of flight trajectory and optimization.
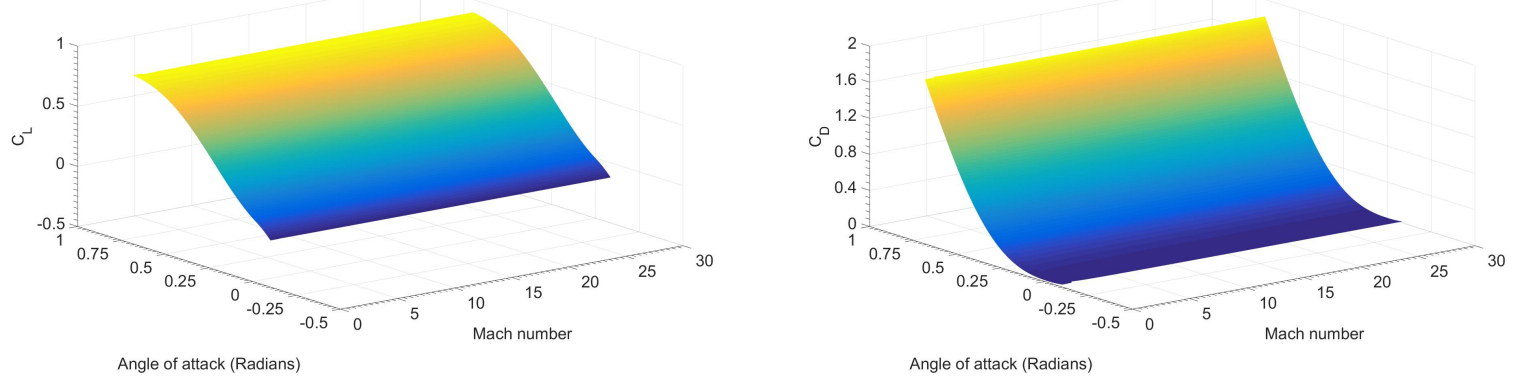

Figure 5. Lift and drag coefficients of the vehicle configuration as a function of Mach number and angle of attack

\section{III.C. Aerothermodynamics}

An engineering level aerothermodynamics model is used to calculate indicative heat fluxes, integrated heat loads and radiative equilibrium temperatures for the purpose of trajectory optimisation. This engineering model is in keeping with initial phase studies and the fidelity of aerodynamic models. Vehicle locations were selected at which heat transfer to the vehicle is calculated along a trajectory. Figure 6 provides a sketch of the monitor points used. A limited number of monitor points were considered for these initial phase studies. Heat transfer is calculated at the nosecone stagnation point and the location of maximum turbulent heating on the nosecone. Heat transfer is also calculated at an acreage monitor point where the heat flux is a function of local surface inclination to the free-stream flow. Heat transfer at a wing leading edge is calculated in addition to heat transfer at a defined distance downstream of the leading edge.

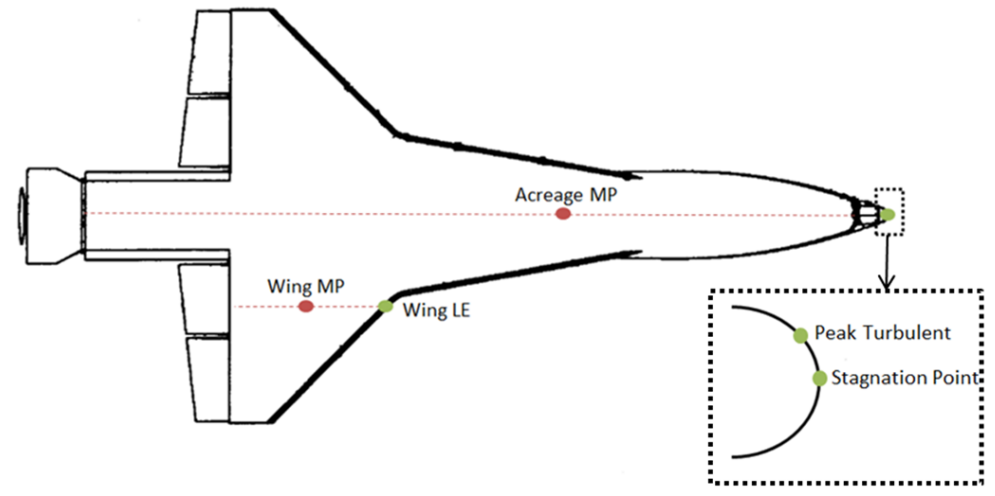

Figure 6. Sketch of monitor point locations on windward side of X-34 geometry

\section{III.C.1. Heat Fluxes and Radiative Equilibrium Temperatures}

The methodology used to calculate heat fluxes at the monitor points shown in Figure 6 is presented in this section. Indicative continuum laminar and turbulent heat transfer coefficients are calculated on the nosecone using the well-known formulation of Detra and Hidalgo ${ }^{16}$ with the application of a suitable equivalent nose radius. This model is modified for the calculation of heat flux at a wing leading edge to take into account the radius of curvature and sweep angle of the wing. The heat flux on the wing a given distance aft of the leading edge is calculated using a flat plate model with angle of attack effects. Acreage heat fluxes are calculated as a function of local surface inclination to the free-stream flow. 
Nosecone Stagnation Point The laminar stagnation point heat flux $q_{s t}\left(\mathrm{~W} / \mathrm{cm}^{2}\right)$ is calculated using Detra and Hidalgos correlation, ${ }^{16}$

$$
q_{s t}=1.135 \sqrt{\frac{\rho_{\infty}}{\rho_{\text {ref }}}}\left(\frac{865}{\sqrt{\frac{2 R_{n}}{0.6096}}}\right)\left(\frac{v_{\infty}}{3048}\right)^{3.15}
$$

where $R_{n}$ is the nose radius, $\rho_{\text {ref }}$ is a reference density defined by Detra and Hidalgo as the density of air at sea level and $\rho_{\infty}$ and $v_{\infty}$ are the free-stream density and velocity, respectively.

Peak Turbulent Nosecone Heating The peak turbulent heat flux on the nose cone is calculated assuming a hemispherical nose of radius $R_{n}$. The turbulent flux $q_{t}$ at a given point can be calculated using Detra and Hidalgos turbulent correlation $\left(\mathrm{W} / \mathrm{cm}^{2}\right)$,

$$
q_{t}=\frac{1.135}{\left(\frac{s}{0.3048}\right)^{0.2}}\left(\frac{\rho_{\infty}}{\rho_{\text {ref }}}\right)^{0.8}\left(\frac{v_{\infty}}{3048}\right)^{3.18} \phi_{t}
$$

where $s$ is the streamlength from the stagnation point to the point of interest and $\phi_{t}$ is a calibration factor accounting for the pressure distribution on the vehicle defined as,

$$
\phi_{t}=\left(\frac{P}{P_{\text {stag }}}\right)^{0.68}\left(1-\left(\frac{P}{P_{\text {stag }}}\right)^{\frac{1}{6}}\right)^{0.4}
$$

where $P_{\text {stag }}$ is the stagnation point pressure. The peak turbulent heat flux corresponds to $\left(P / P_{\text {stag }}\right)=0.57$ (see Detra and Hidalgo ${ }^{16}$ ) which assuming a Newtonian pressure distribution and a hemispherical nose allows the streamlength $s$ corresponding to the location of peak turbulent heating to be calculated.

The corresponding laminar heat flux at the point of peak turbulent heat flux can be approximated by, ${ }^{17}$

$$
q_{l}=q_{s t} \cos ^{\frac{3}{2}} \gamma
$$

where $\gamma$ is the angle from the nose centreline. The peak turbulent heat flux is then taken to be, ${ }^{17}$

$$
q_{\text {tmax }}=\max \left(q_{t}, q_{l}\right)
$$

Wing LEAding EDGE A simplified model for the heat flux at the wing leading edges, taking account of radius of curvature and sweep angle, is taken from. ${ }^{17}$ The wing leading edges are assumed to be exposed directly to the free stream to provide indicative fluxes. This condition is more likely to be satisfied at higher Mach numbers but is also configuration and attitude dependent. Sweeping a wing or leading edge of a vehicle will generally result in a reduction in the convective heat flux at the surface. An effective angle of sweep $\Lambda_{e}$ can be defined as a function of sweep angle $\Lambda$ and angle of attack $\alpha$ as, ${ }^{17}$

$$
\Lambda_{e}=\sin ^{-1}(\sin \Lambda \cos \alpha)
$$

The ratio of swept $q_{\Lambda}$ to non-swept heat flux $q_{0}$ is shown ${ }^{17}$ to be,

$$
\frac{q_{\Lambda}}{q_{0}}=0.755 \cos \Lambda_{e}
$$

valid in the approximate range $0 \mathrm{deg}<\Lambda<60 \mathrm{deg}$. The relief factor of 0.755 accounts for the cylindrical geometry of the wing leading edge.

Wing Monitor Point An estimate of heating on the wings away from the leading edge or stagnation point is calculated using expressions taken from SAE. ${ }^{17}$ The wing is approximated as a flat plate at angle of attack $\alpha$ with the heat flux a distance from the leading edge based on the state of the boundary layer for laminar:

$$
q_{w, x}=q_{s t}(x) \frac{0.0312}{1.068} \alpha^{\frac{2}{3}}
$$

and turbulent:

$$
q_{w, x}=q_{t}(x) \frac{0.333}{5.0} \alpha
$$


where $q_{s t}(x)$ is the Detra-Hidalgo equation for stagnation point heating in Eq. (9) evaluated at a nose radius of $x$. Similarly, $q_{t}(x)$ is the Detra-Hidalgo equation for peak turbulent heating in Eq. (10) evaluated at a nose radius of $x$. These equations are applicable far downstream from the leading edge (greater than approximately 10 leading edge radii from the leading edge). They can be used for preliminary analysis of aerodynamic heating but are not recommended for more detailed work. Hot wall corrections can be applied to these expressions but have not been included for simplicity. These corrections are generally small when the freestream total enthalpy is large.

Acreage Monitor Point It is not appropriate to approximate the acreage as a flat plate as is done for the wings. Instead, the heat flux at a point on the acreage $q_{a c r}$ is calculated based on the modified Lees method,

$$
q_{a c r}=q_{s t} k_{1}\left(k_{2}+\left(1-k_{2}\right) \sin ^{k_{3}} \theta\right)
$$

where $\theta$ is the angle between the local surface and the free-stream flow and $k_{1}, k_{2}$ and $k_{3}$ are constants that must be calibrated. For initial phase studies, these constants were calibrated using a boundary layer solution at a point $1.5 \mathrm{~m}$ downstream of the nosetip on the windward streamline (mid-nosecone). The vehicle angle of attack can be accounted for in the value of $\theta$. This expression is intended to be used for preliminary analysis, However, the constants can be calibrated to higher fidelity predictions (boundary layer solutions for example) using the concept geometry. Hence, heat flux predictions can be easily adjusted through the three constants as the fidelity of future modelling increases.

Free Molecular Heat Flux and Bridging Free molecular heating $q_{f m}$ is approximated in the limit of infinite speed ratio with complete thermal accommodation (which for the stagnation point is simply the incoming kinetic energy flux),

$$
q_{f m}=\frac{1}{2} \rho_{\infty} v_{\infty}^{3} \sin \theta
$$

Accounting for the effects of finite speed ratio, varying thermal accommodation coefficient and varying temperature ratio (see e.g., Schaaf ${ }^{18}$ ) can easily be calculated, but would introduce a level of detail that is not justified at this stage of the study.

For simplicity, the applied heat flux is taken to be the minimum selected from the continuum $q_{\text {cont }}$ and free-molecular formulations at each point on the trajectory. This is justified for early design phase studies since it provides a conservative heat load when compared to more sophisticated Knudsen based bridging techniques. Hence, at any given trajectory point:

$$
q=\min \left(q_{\text {cont }}, q_{f m}\right)
$$

Radiative Equilibrium Temperature Radiative equilibrium wall temperatures are calculated by balancing the heat radiated away from the vehicles surface with the wall temperature corrected heat fluxes impinging on the surface. The variation of heat flux with wall temperature is approximated by using the enthalpy based film coefficient,

$$
q_{h o t}=q_{c} \frac{h_{0}-h_{w}}{h_{0}-h_{300}}
$$

where $q_{c}$ is the cold wall heat flux (calculated using the aforementioned methods), $h_{w}$ is the wall enthalpy, $h_{300}$ is air enthalpy at a temperature of $300 \mathrm{~K}$ and $h_{0}$ is the total enthalpy defined as,

$$
h_{0}=h_{\infty}+\frac{1}{2} v_{\infty}^{2}
$$

Both $h_{w}$ and $h_{300}$ are calculated from real gas tables for air generated using the NASA Glenn CEA code. ${ }^{19}$ The radiative heat flux away from the vehicles surface is calculated using the Stefan-Boltzmann law with an appropriate emissivity $\epsilon$,

$$
q_{\text {rerad }}=\sigma \epsilon T_{w}^{4}
$$

where $\sigma$ is the Stefan-Boltzmann constant. The radiative equilibrium temperature is the wall temperature $T_{e q}=T_{w}$ for which,

$$
q_{\text {hot }}\left(T_{w}\right)-q_{\text {rerad }}\left(T_{w}\right)=0
$$

This equation is solved with a numerical root finding algorithm. 
Heat fluxes are calculated for each trajectory time-step at the nose stagnation point from Eq. (9)), location of peak turbulent heating on the nosecone from Eq. (10)), wing leading edge in Eq. (15)), wing monitor point in Eqs. (16)-(17)), and acreage monitor point in Eq. (18)). The radiative equilibrium temperature corresponding to heat fluxes at each monitor point can be calculated either within the optimisation, or through post-processing. For the design trade-off and feasbility studies, to improve the computational run time, only the heat fluxes were calculated inside the optimisation.

\section{III.D. Propulsion}

The rocket engines are modelled using standard Tsiolkovsky rocket equations, with configurable inputs specifying the specific impulse $I_{s p}$ and thrust $F_{T_{v a c}}$ in a vacuum. A throttle control $\tau \in[0,1]$ is added that dictates the fraction of maximum available thrust applied and fuel mass flow (and therefore fuel consumption). A simplifying assumption is made that the mass flow varies linearly with thrust. The applied thrust and mass flow rate per engine are then calculated as,

$$
\begin{aligned}
\frac{d m_{p}}{d t} & =\dot{m}_{p}=\tau n_{e n g} n_{n o z z} \frac{F_{T_{v a c}}}{g_{0} I_{s p}} \\
F_{T}(h) & =F_{T}=\tau n_{\text {eng }} n_{n o z z}\left(F_{T_{v a c}}-p_{a t m} A_{e}\right)
\end{aligned}
$$

where $n_{n o z z}$ are the number of nozzles per engine, and $n_{\text {eng }}$ number of engines on the vehicle. A penalty proportional to atmospheric pressure $p_{a t m}$ and nozzle exit area $A_{e}$ is introduced to account for the difference in nozzle expansion under pressure compared to in a vacuum.

The two main stage engines uses a LOX/Kerosene propellant with an $I_{s p}$ between 300-400 s, based on the Yuzhnoye RD-8 series of rocket engines. The number and rating of engines are determined through the design trade-off studies accounting for engine designs currently at TRL 7-9 (i.e., that are either currently available, or predicted to be available in the next 5 years).

\section{III.E. Environment}

The Earth is modelled as an oblate spheroid based on the WSG-84 model. The gravitational field was modelled using $4^{\text {th }}$ order spherical harmonics (accounting for $J_{2}, J_{3}$ and $J_{4}$ terms) for accelerations in the radial $g_{r}$ and transverse $g_{\phi}$ directions. ${ }^{20}$ The angular rotation of the Earth is assumed constant at $\omega_{E}=7.292115 \times 10^{-5} \mathrm{rad} / \mathrm{s}$.

The atmospheric conditions - temperature $T_{a t m}$, pressure $p_{a t m}$, density $\rho_{a t m}$ and speed of sound - are modelled using the Standard US-76 global static atmospheric model extended up to an altitude of $1000 \mathrm{~km}$ above the Earth surface. ${ }^{21}$

\section{III.F. Flight dynamics and control}

A 3-DOF variable point mass dynamic model is used where the spaceplane is a time-varying mass located at the centre-of-gravity of the vehicle. The state vector for the flight dynamics $\mathbf{x}_{d y n}=[\mathbf{r}, \dot{\mathbf{r}}]$ is the spherical coordinates for the position $\mathbf{r}=[r, \lambda, \theta]$ and the velocity $\dot{\mathbf{r}}=[v, \gamma, \chi]$ where $r$ is the radial distance, $(\lambda, \theta)$ are the latitude and longitude, $v$ is the magnitude of the relative velocity vector directed by the flight path angle

$\gamma$ and the flight heading angle $\chi$. The equations of motion are expressed in the Earth-Centred-Earth-Fixed rotating reference frame. ${ }^{22,20}$

$$
\begin{aligned}
& \dot{h}=\dot{r}=v \sin \gamma \\
& \dot{\lambda}=\frac{v \cos \gamma \sin \chi}{r} \\
& \dot{\theta}=\frac{v \cos \gamma \cos \chi}{r \cos \lambda}
\end{aligned}
$$




$$
\begin{aligned}
\dot{v}= & \frac{F_{T} \cos (\alpha+\varepsilon)-D}{m}-g_{r} \sin \gamma+g_{\phi} \cos \gamma \cos \chi+\omega_{\mathrm{E}}^{2} r \cos \lambda(\sin \gamma \cos \lambda-\cos \gamma \sin \chi \sin \lambda) \\
\dot{\gamma}= & \frac{F_{T} \sin (\alpha+\varepsilon) \cos \mu+L}{m v}-\frac{g_{r}}{v} \cos \gamma-\frac{g_{\phi}}{v} \sin \gamma \cos \chi+\frac{v}{r} \cos \gamma+2 \omega_{\mathrm{E}} \cos \chi \cos \lambda \\
& \quad+\omega_{\mathrm{E}}^{2}\left(\frac{r}{v}\right) \cos \lambda(\sin \chi \sin \gamma \sin \lambda+\cos \gamma \cos \lambda) \\
\dot{\chi}= & \frac{L \sin \mu}{m v \cos \gamma}-g_{\phi} \sin \chi-\left(\frac{v}{r}\right) \cos \gamma \cos \chi \tan \lambda+2 \omega_{\mathrm{E}}(\sin \chi \cos \lambda \tan \gamma-\sin \lambda) \\
& -\omega_{\mathrm{E}}^{2}\left(\frac{r}{v \cos \gamma}\right) \cos \lambda \sin \gamma \cos \chi
\end{aligned}
$$

where $m$ is the time-varying mass of the vehicle, $\varepsilon$ is the pitch offset angle between the direction of thrust $F_{T}$ and the longitudinal plane of the vehicle, $\left[g_{r}, g_{\phi}\right]$ are the gravitational accelerations in the radial and transverse directions, and $L$ and $D$ are the aerodynamic lift and drag forces, respectively.

The trajectory dynamics are controlled by adjusting the thrust vector. The magnitude of the thrust and mass flow applied is controlled by $\tau$, and the direction through the angle of attack $\alpha$, thrust offset angle $\varepsilon$ and the bank angle $\mu$. The engines are assumed fixed with no gimbled thrust at this stage, thus the control law also dictates the partial attitude of the vehicle.

\section{Optimisation}

In this section, the general formulation is presented for trajectory and design optimisation of the conceptual design. The optimisation seeks to find a mission flight profile that minimises the propellant usage, subject to a number of vehicle loading and thermal constraints, and a set of design parameters that both minimise the required gross vehicle mass and maximise the downrange distance while being able to meet the target mission.

The first step was to formulate the problem as an optimal control problem: given the system dynamics for the chosen vehicle configuration, full or partial boundary conditions for the initial and final states of the vehicle and any path constraints, the aim is to find a optimal control law that minimises a given performance index, and to analyse trade-offs between different configurations.

The mission is decomposed into a number of user-defined phases, with different system models, objectives and constraints used within each phase. The phase decomposition is also used to accommodate discontinuities within the system and performance models, such as separating the sub-, trans- and super/hypersonic aerodynamic models, or for vehicle staging.

A direct multi-shooting transcription method is then employed to transform the continuous optimal control problem into a non-linear programming problem (NLP). The NLP is then solved with a gradient based optimisation algorithm using a multi-start approach to generate first-guess solutions.

\section{IV.A. Optimal control problem formulation}

Optimal control problems can be formulated as:

$$
\begin{aligned}
& \min _{\mathbf{u} \in U} J \\
& \text { s.t. } \\
& \dot{\mathbf{x}}=\mathbf{F}(\mathbf{x}, \mathbf{u}, t) \\
& \mathbf{g}(\mathbf{x}, \mathbf{u}, t) \geq 0 \\
& \boldsymbol{\psi}\left(\mathbf{x}_{0}, \mathbf{x}_{f}, t_{0}, t_{f}\right) \geq 0 \\
& t \in\left[t_{0}, t_{f}\right]
\end{aligned}
$$

where $J$ is a function of the state vector $\mathbf{x}:\left[t_{0}, t_{f}\right] \rightarrow \mathbb{R}^{n}$, control vector $\mathbf{u} \in L^{\infty}$ and time $t, \mathbf{F}$ is a set of differential equations describing the dynamics of the system, $\mathbf{g}$ is a set of algebraic inequalities describing path constraints and $\boldsymbol{\psi}$ is a set of algebraic inequalities describing boundary constraints.

The optimal control problem is transcribed into a nonlinear programming problem by using a multiphase, multiple-shooting approach. The mission is initially divided into $n_{p}$ user-defined phases. Within each 
phase, the time interval is further divided into $n$ multiple shooting segments.

$$
\cup_{k=1}^{n_{p}} \cup_{i=0}^{n-1}\left[t_{i, k}, t_{i+1, k}\right]
$$

The trajectory is numerically integrated within the interval $\left[t_{i, k}, t_{i+1, k}\right]$ with initial conditions $\mathbf{x}_{i, k}$. Within each interval $\left[t_{i, k}, t_{i+1, k}\right]$, the control is further discretised into $n_{c}$ control nodes $\left\{u_{0}^{i, k}, \ldots, u_{n_{c}}^{i, k}\right\}$ and collocated on Tchebycheff points in time.

Continuity constraints on the control and states can be imposed between each shooting element, and between phases such that

$$
\begin{aligned}
& \left.\begin{array}{l}
\mathbf{x}_{i, k}=F\left(\left[t_{i-1, k}, t_{i, k}\right], \mathbf{x}_{i-1, k}\right) \\
\mathbf{u}_{n_{c}}^{i-1, k}=\mathbf{u}_{0}^{i, k}
\end{array}\right\} \text { for } k=1, \ldots, n_{p} \text { and } i=2, \ldots, n \\
& \left.\begin{array}{l}
\mathbf{x}_{1, k}=\mathbf{x}\left(t_{n+1, k-1}\right) \\
\mathbf{u}_{0}^{1, k}=\mathbf{u}_{n_{c}}^{n+1, k-1}
\end{array}\right\} \text { for } k=2, \ldots, n_{p}
\end{aligned}
$$

where $F\left(\left[t_{i-1, k}, t_{i, k}\right], \mathbf{x}_{i-1, k}\right)$ is the final state of the numerical integration on the interval $\left[t_{i-1, k}, t_{i, k}\right]$ with initial conditions $\mathbf{x}_{i-1, k}$. This approach increases the degrees of freedom of the optimisation process reducing the sensitivity of the overall problem to its variables although at a cost of a steep increase in the number of optimisation variables.

The trajectory optimisation vector is therefore composed of:

- control nodes of each shooting segment $\left\{\mathbf{u}_{0}^{i, k}, \ldots, \mathbf{u}_{n_{c}}^{i, k}\right\}$ for for $i=1, \ldots, n$ and $k=1, \ldots, n_{p}$,

- time of flight for each shooting segment $\Delta t_{k}$ for $k=1, \ldots, n_{p}$,

- initial state and control variables of each shooting segment within every phase that should be matched with the previous segment or phase $\mathbf{x}_{1, k}$ and $\mathbf{u}_{0}^{1, k}$ for $k=2, \ldots, n_{p}$.

\section{IV.B. Single objective optimisation algorithm}

Problem (28) was solved with Matlab optimiser fmincon using the interior point algorithm, which is a gradient based local solver for the solution of single objective NLP with nonlinear constraints.

A multi-start strategy was used to generate a set of first guess solution vectors that were randomly initialised within the defined search space through Latin Hypercube Sampling. This allowed a better exploration of the search space and reduces the sensitivity of system to the first guess values. Integration of system of ODEs in Eqs. (26) was performed with a fixed step Bogacki-Shampine Runge-Kutta method of third order.

\section{IV.C. Multidisciplinary design optimisation}

A multidisciplinary design optimisation (MDO) approach was used to study the optimality of certain design and operational parameters of the vehicle. These design optimisation parameters were added to the trajectory optimisation vector to be determined by the optimiser.

The mission flight path starts just after the separation of the vehicle from the carrier aircraft, therefore the initial state vector of the spaceplane is dependant on the state of the carrier aircraft. A conservative estimation was made for the maximum altitude and velocity (or Mach number) that could be achieved by the carrier aircraft at separation. Two nominal geographic points (latitude and longitude) were selected accounting for range of the carrier aircraft, and safety/regulatory criteria. The flight path and heading angle were set as optimisation design variables, with upper and lower bounds set to allow for the limitations of the separation manoeuvre.

Static design parameters were added to size the engines for each stage, and the wing area for the returnable, reusable first stage. The overall objective was the minimisation of the gross vehicle mass subject to the nominal design mission which included a target orbit and payload mass, and an unpowered downrange return. This choice of objective required that each of the design choices directly or indirectly affect the mass of the vehicle. The system of parametric mass estimating relationships (MER) in Section III.A were defined relative to these design variables. For this study, the mass and sizing of the thermal protection system (TPS) was not included directly in the design optimisation loop, though later studies will examine the requirements for limits on heat load and temperatures based on different TPS. 
The propulsion system were sized based on optimising the total mass of propellants for each stage and scaling factors on the maximum vacuum thrust rating for the engines. The mass of the propellant was used to determine the volume and mass of the tanks, while the vacuum rating was used to scale the mass of the engine and engine structure. The engines were scaled relative to two nominal LOX-Kerosene rocket engines manufactured by Yuzhnoye Design Office: the first stage has a main engine with a vacuum thrust of 88.4 tf, vacuum $I_{s p}$ of $332 \mathrm{~s}$ and a mass of $1280 \mathrm{~kg}$. The second stage uses the RD-809K engine, with a vacuum thrust of $10 \mathrm{tf}$, vacuum $I_{s p}$ of $352 \mathrm{~s}$, and a mass of $330 \mathrm{~kg}$.

The sizing of the aerodynamic surfaces is another key design parameter for the vehicle, here through the wing area. As the ascent is rocket-based, with a high thrust force compared to the lift, the ascent drives the design to small wing areas to reduce drag (not accounting for any stability or control surface requirements). The requirement for a glided return to some coastal site relatively in-plane to the trajectory, drives up the wing area to improve the down or cross ranges achievable. The aerodynamic model for the coefficients is assumed constant for all design options, with the wing reference area $S_{\text {wing }}$ scaled relative to the total reference area $S_{r e f}$. The lift force $L$ is calculated based on,

$$
\begin{aligned}
C_{L, m d o} S_{r e f} & =C_{L, w i n g} S_{w i n g}+C_{L, i} S_{i} \\
L & =\frac{1}{2} \rho v_{\infty}^{2} C_{L, m d o} S_{r e f}
\end{aligned}
$$

where $C_{L, i}, S_{i}$ are the coefficients of lift and corresponding reference area for the unchanged components of the fuselage, fairing and tail. The wing reference area $S_{\text {wing }}$ is scaled relative to the nominal design value.

In this study, the downrange distance is maximised assuming no cross-range (i.e., the trajectory is entirely in-plane). This is used as a figure of merit for the capabilities of the system assuming no specific landing sites are given, and assuming no requirements for a return to landing site. This is consistent with the commercial drivers for the system that prioritised global operation and flexibility.

\section{Analysis and results}

In the following, three different scaling factors for the wing reference area are analysed: $60 \%, 100 \%$ and $120 \%$ of $S_{\text {wing }}$. Two release points were chosen, both west of the UK to minimise the time the atmospheric trajectory was over any populated land. The first assumes a north-west flight departing from Prestwick airport in Scotland $\left(58.806^{\circ} \mathrm{N}, 12.747^{\circ} \mathrm{W}\right)$, the second is north-north-east of Faroe Islands $\left(63.040^{\circ} \mathrm{N}, 6.378^{\circ} \mathrm{W}\right)$. The initial flight path angle after separation is an optimisation variable with a constraint $\gamma_{0} \leq 20^{\circ}$. Path constraints are added on the normal and axial accelerations such that $\left|a_{x}(t)\right|,\left|a_{z}(t)\right| \leq 6 g_{0}$.

The ascent was optimised based on the objective function,

$$
\min _{\mathbf{p} \in \mathbb{D}}\left(m_{\text {gross }}\right)
$$

where the gross vehicle mass is the sum of the dry and fuel masses of Stage 1 and Stage 2, plus the payload mass. The optimisation vector $\mathbf{p}$ contains: the 4 vehicle design variables (vacuum thrust scaling factors for Stage 1 and 2, total fuel mass for Stage 1 and 2), the initial flight path $\gamma_{0}$ and heading angle $\chi_{0}$ just after carrier separation, and the multi-phase, multi-shooting trajectory optimisation vector $\mathbf{c}$ listed in Section IV.A. Figure 7 shows the different user-defined phases of the mission, and the relation to vehicle staging.

The atmospheric descent was optimised based on the objective function maximising the central angle of the descent range based on the start and end points of the atmospheric re-entry phase (Phase 4),

$$
\max _{\mathbf{c} \in \mathbb{C}}\left(\frac{d_{\text {gnd }}}{r_{\mathrm{E}}(\lambda=0)}\right)
$$

where the descent range $d_{g n d}$ is given by the Haversine equation,

$$
d_{g n d}=2 r \arcsin \left(\sqrt{\text { hav } \Delta \lambda_{P h 4}+\cos \lambda_{P h 4,0} \cos \lambda_{P h 4, f} \text { hav } \Delta \theta_{P h 4}}\right)
$$

Table 1 summarises the initial and final conditions, the objective function, constraints, number of phases, elements and relevant settings for the ascent trajectory, which was broken into two phases to model stage separation. For each of the 6 cases, the multi-start algorithm was used to generate 35 different first guess solutions. 
Table 1. Mission and optimisation parameters for the ascent trajectory

\begin{tabular}{|c|c|}
\hline Initial conditions & $\begin{array}{l}\text { Altitude } h=12192 \mathrm{~m} \\
\text { Velocity } v=206.55 \mathrm{~m} / \mathrm{s}(\text { Mach }=0.7) \\
\text { Latitude } \lambda=58.805820^{\circ} \mathrm{N}(\text { Prestwick }), 63.039952^{\circ} \mathrm{N}(\text { Faroe }) \\
\text { Longitude } \theta=-12.747062^{\circ} \mathrm{E}(\text { Prestwick }),-6.378264^{\circ} \mathrm{E}(\text { Faroe }) \\
\text { Flight path angle } \gamma \leq-20^{\circ}\end{array}$ \\
\hline Final orbit & Semimajor axis $a=R_{E}+600 \mathrm{~km}$, eccentricity $e=0$, inclination $i=88.2^{\circ}$ \\
\hline Number of phases & 2 \\
\hline Elements per phase & 1 \\
\hline Control nodes per element & 6 \\
\hline $\begin{array}{l}\text { Bounds on state } \\
\text { optimisation variables } \\
\text { (Stage } 1 / \text { Phase } 1 \text { ) }\end{array}$ & $\begin{array}{l}10 \mathrm{~km} \leq h \leq 250 \mathrm{~km} \\
20 \mathrm{~m} / \mathrm{s} \leq v \leq 5000 \mathrm{~m} / \mathrm{s} \\
-10^{\circ} \leq \gamma \leq 20^{\circ} \\
-20^{\circ} \leq \chi \leq 60^{\circ} \\
-90^{\circ} \leq \lambda \leq 90^{\circ} \\
-180^{\circ} \leq \theta \leq 180^{\circ} \\
m_{\text {dry }, \text { S1 }} \leq m \leq m_{\text {gross }, \text { veh }} \\
120 \mathrm{~s} \leq \text { tof } \leq 900 \mathrm{~s}\end{array}$ \\
\hline $\begin{array}{l}\text { Bounds on state } \\
\text { optimisation variables } \\
\text { (Stage } 2 / \text { Phase } 2 \text { ) }\end{array}$ & $\begin{array}{l}30 \mathrm{~km} \leq h \leq 1000 \mathrm{~km} \\
2000 \mathrm{~m} / \mathrm{s} \leq v \leq 10000 \mathrm{~m} / \mathrm{s} \\
-60^{\circ} \leq \gamma \leq 90^{\circ} \\
-20^{\circ} \leq \chi \leq 60^{\circ} \\
-90^{\circ} \leq \lambda \leq 90^{\circ} \\
-180^{\circ} \leq \theta \leq 180^{\circ} \\
m_{d r y, S 2} \leq m \leq\left(m_{\text {gross }, \text { veh }}-m_{\text {dry }, S 1}-m_{\text {fuel }, S 1}\right) \\
120 \mathrm{~s} \leq \text { tof } \leq 600 \mathrm{~s}\end{array}$ \\
\hline $\begin{array}{l}\text { Bounds on trajectory control } \\
\text { optimisation variables } \\
(\text { Phase } 1)\end{array}$ & $\begin{array}{l}0 \leq \delta_{\tau} \leq 1 \\
-5^{\circ} \leq \alpha \leq 40^{\circ} \\
-15^{\circ} \leq \mu \leq 15^{\circ}\end{array}$ \\
\hline $\begin{array}{l}\text { Bounds on trajectory controls } \\
\text { optimisation variables } \\
\text { (Phase } 2)\end{array}$ & $\begin{array}{l}0 \leq \tau \leq 1 \\
-10^{\circ} \leq \alpha \leq 60^{\circ} \\
0^{\circ} \leq \mu \leq 0^{\circ}\end{array}$ \\
\hline Path constraints & $\begin{array}{l}\text { Axial acceleration: }\left|a_{x}\right| \leq 6 g_{0} \\
\text { Normal acceleration: }\left|a_{z}\right| \leq 6 g_{0}\end{array}$ \\
\hline
\end{tabular}




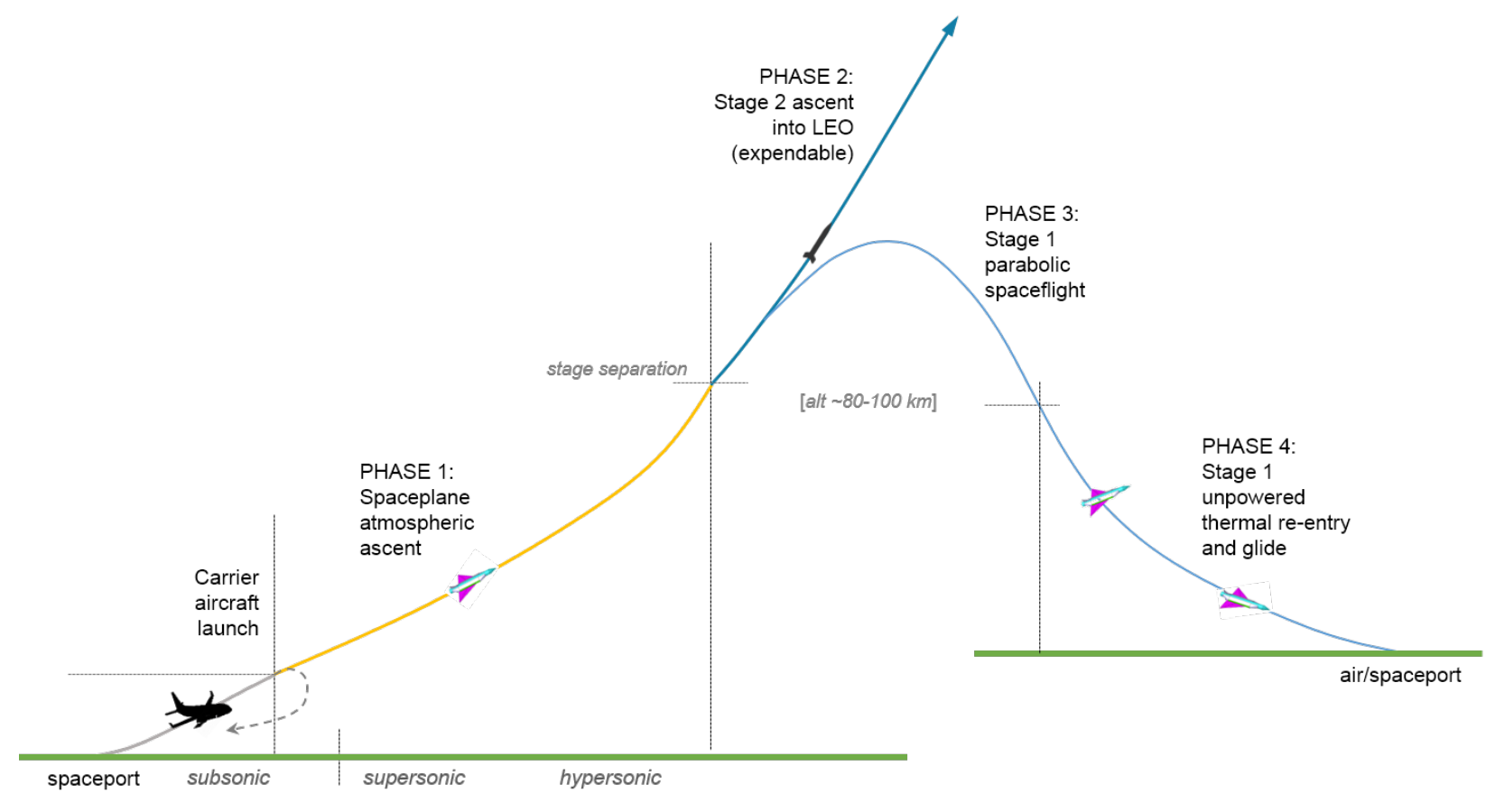

Figure 7. Illustration showing user-defined mission phases

The re-entry trajectory was broken into 2 phases. The first phase (Phase 3 ) is the trajectory arc between the separation point of the two stages and the atmospheric re-entry, here defined to start at an altitude of $80 \mathrm{~km}$. In that high altitude phase, the trajectory is ballistic due to the absence of significant atmospheric density and thrust. As such there is no need to derive an optimal control law based on vehicle attitude; this phase was excluded from the optimisation and simply propagated forward in time until the descent altitude reached the set limit. The second phase, Phase 4, is controllable with aerodynamic surfaces, and was thus optimised. Relevant parameters are reported in Table 2.

The optimised vehicle design parameters are given in Table 3 based on estimates for a composite material structure. Table 4 gives the optimal initial conditions for the ascent trajectory, and Table 5 reports the optimised values for the approach to landing of Stage 1, including the maximised downrange distances.

As expected, higher wing areas generally resulted in higher dry masses, propellent masses and engine sizes for each stage. An exception is the second stage for the nominal wing area $\left(1.0 S_{\text {wing }}\right)$ departing from Prestwick. While the gross vehicle mass for this case is between the gross masses for the smaller and larger wing areas, as expected, the sizings for each stage differs. The optimiser found a solution with a larger first stage, very similar to that of the $1.2 S_{\text {wing }}$ case for both engine sizing and mass, and a lighter second stage with a smaller engine. This combination gave the longest downrange distance as a larger first stage means a higher velocity at stage separation, longer ballistic phase and hence better downrange distance. This is also evident from Fig. 10(e) that shows this case has the highest $\mathrm{T} / \mathrm{W}$ ratio.

Table 3 also lists the residual or remaining fuel mass, which ranges between 0.0045-0.13 kg (equivalent to a factor $10^{-4}$ of the propellant mass). The problem formulation requires the total on-board fuel mass to be estimated prior to the trajectory optimisation in order to determine the vehicle masses. The optimisation of each trajectory within the design optimisation cycle minimises the fuel usage, with the target that all the fuel onboard is consumed during flight.

Figures 8 and 9 show the optimal trajectories for the nominal wing area for the two different release points. The trajectories are shown for all 4 phases (as illustrated conceptually in Fig. 7). While the trend of the two trajectories are the same, and both fulfill the mission requirements, the absolute values for the descent are different. This highlights the competing objectives finding the minimal gross vehicle mass in order to inject $500 \mathrm{~kg}$ into the target orbit, and the maximal downrange distance. The two locations are relatively close together on the Earth, separated by surface distance of $582.6 \mathrm{~km}$. The maximised downrange achievable departing from Prestwick is, on average, $224 \mathrm{~km}$ more then Faroe. With the cases for Faroe 
Table 2. Mission and optimisation parameters for atmospheric descent trajectory

\begin{tabular}{ll}
\hline Initial conditions & $\mathbf{x}\left(t_{0, P 4}\right)=\mathbf{x}\left(t_{f, P 3}\right)$ \\
& $\alpha\left(t_{0, P 4}\right)=40^{\circ}, \mu\left(t_{0, P 4}\right)=0^{\circ}$ \\
& $m(t)=m_{d r y, S 2}$ \\
& $h\left(t_{f, P 4}\right) \leq 1 \mathrm{~km}$ \\
& $\gamma\left(t_{f, P 4}\right) \geq-20^{\circ}$ \\
\hline Final conditions & 1 \\
& 2 \\
\hline Number of phases & 6 \\
Elements per phase & $10 \mathrm{~m} \leq h \leq 100 \mathrm{~km}$ \\
Control nodes per element & $20 \mathrm{~m} / \mathrm{s} \leq v \leq 5000 \mathrm{~m} / \mathrm{s}$ \\
\hline Bounds on state & $-60^{\circ} \leq \gamma \leq 60^{\circ}$ \\
optimisation variables & $-20^{\circ} \leq \chi \leq 60^{\circ}$ \\
(Phase 4) & $-90^{\circ} \leq \lambda \leq 90^{\circ}$ \\
& $-180^{\circ} \leq \theta \leq 180^{\circ}$ \\
& $120 \mathrm{~s} \leq \mathrm{t} \leq 1200 \mathrm{~s}$ \\
\hline Bounds on trajectory control & $\tau=0($ unpowered) \\
optimisation variables & $-5^{\circ} \leq \alpha \leq 40^{\circ}$ \\
(Phase 4) & $-15^{\circ} \leq \mu \leq 15^{\circ}$ \\
\hline Path constraints & Axial acceleration: $\left|a_{x}\right| \leq 6 g_{0}$ \\
& Normal acceleration: $\left|a_{z}\right| \leq 6 g_{0}$ \\
\hline
\end{tabular}

having a lighter weight configuration to reach orbit, on average 2.3 tonnes. For all wing areas, the Prestwick location required a larger mass vehicle to reach orbit, which gave a longer downrange, while the cases from Faroe were the opposite.

Figure 10 shows the trajectories for the Stage 1+2 combined ascent (Phase 1), followed by the Stage 1 ballistic coast after stage separation (Phase 3), and the Stage 1 atmospheric re-entry (Phase 4) for the 3 different wing areas studied. This shows the trade-off of increasing wing area, where increasing the aerodynamic contribution of wing can increase the glide performance of the vehicle, it is at the expense of increased dry mass. The net effect shows an optimal configuration somewhere near the nominal wing reference area, looking only at the descent performance.

\section{Conclusion}

This paper presented a conceptual design and performance analysis of a partially re-usable space launch vehicle for small payloads. The system was designed for a nominal mission of delivering a $500 \mathrm{~kg}$ payload to a circular $600 \mathrm{~km}, 88.2^{\circ}$ polar orbit. The aim of the system design was to develop a commercially viable launch system for near-term operation, thus emphasis is placed on the efficient use of high TRL technologies. The final design employed a multi-stage, rocket-based spaceplane air-launched from a carrier aircraft. The first stage is fully recoverable through an unpowered glided descent to a secondary landing site. Stage separation occurs around $70 \mathrm{~km}$, with the second expendable stage reaching the nominal mission orbit.

A multidisciplinary design optimisation on the system configuration was run to size the engines of both stages and the Stage 1 wing area. The system had to meet two objectives: to minimise the gross vehicle mass, and to maximise the downrange. Test cases were run for two different geographic release points off the UK west coast, and for 3 different wing areas relative to the nominal aerodynamic $S_{\text {wing }}$. All 6 test cases are capable of meeting all the mission requirements. The gross masses range between $65-72$ tonnes, and the downrange between $716-1343 \mathrm{~km}$. The best downrange was achieved with the nominal wing reference area departing off the coast of Prestwick, with a gross vehicle mass of 70.87 tonnes and a downrange of $1343 \mathrm{~km}$. 
This configuration had a comparatively larger first stage with an engine vacuum thrust rating of $1164 \mathrm{kN}$ and dry mass of $11343 \mathrm{~kg}$, and a second stage with an engine vacuum thrust rating of $10.6 \mathrm{kN}$ and dry mass of $1852.6 \mathrm{~kg}$.

Table 3. Optimal vehicle design parameters (for a fixed payload mass of $500 \mathrm{~kg}$ )

\begin{tabular}{|c|c|c|c|c|c|c|c|}
\hline & \multirow[b]{2}{*}{ Wing area: } & \multicolumn{3}{|c|}{ Prestwick } & \multicolumn{3}{|c|}{ Faroe } \\
\hline & & $0.6 S_{\text {wing }}$ & $S_{\text {wing }}$ & $1.2 S_{w i n g}$ & $0.6 S_{w i n g}$ & $S_{\text {wing }}$ & $1.2 S_{w i n g}$ \\
\hline \multirow[t]{4}{*}{ Stage 1: } & Vacuum thrust $(\mathrm{kN})$ & 1112.6 & 1164.3 & 1170.6 & 1050.1 & 1097.1 & 1123.9 \\
\hline & Propellant mass (tonne) & 43.628 & 45.87 & 45.957 & 39.276 & 41.094 & 43.814 \\
\hline & Dry mass (tonne) & 10.665 & 11.343 & 11.635 & 10.638 & 11.304 & 11.535 \\
\hline & Residual mass (kg) & 0.13458 & 0.12949 & 0.13277 & 0.036833 & 0.036644 & 0.14578 \\
\hline \multirow[t]{5}{*}{ Stage 2: } & Vacuum thrust (kN) & 139.17 & 129.61 & 140.28 & 139.03 & 143.31 & 151.87 \\
\hline & Propellant mass (tonne) & 10.96 & 10.643 & 11.258 & 12.089 & 12.505 & 12.778 \\
\hline & Dry mass (tonne) & 1.8863 & 1.8526 & 1.898 & 1.931 & 1.9572 & 1.9706 \\
\hline & Residual mass (kg) & 0.016405 & 0.016636 & 0.016187 & 0.0044781 & 0.0013427 & 0.017646 \\
\hline & Vehicle gross mass (tonne) & 68.307 & 70.872 & 71.914 & 64.98 & 67.899 & 71.279 \\
\hline
\end{tabular}

Table 4. Optimal initial conditions just after release point from carrier aircraft

\begin{tabular}{ccccccc}
\hline & \multicolumn{3}{c}{ Prestwick } & \multicolumn{3}{c}{ Faroe } \\
Wing area: & $0.6 S_{\text {wing }}$ & $S_{\text {wing }}$ & $1.2 S_{\text {wing }}$ & $0.6 S_{\text {wing }}$ & $S_{\text {wing }}$ & $1.2 S_{\text {wing }}$ \\
\hline Flight path angle $\gamma\left(t_{0}\right)(\mathrm{deg})$ & 9.47 & 10.81 & 7.06 & 12.44 & 9.18 & 7.17 \\
Flight heading angle $\chi\left(t_{0}\right)(\mathrm{deg})$ & 0.15 & 0.12 & 0.09 & 0.90 & 0.80 & 0.73 \\
\hline
\end{tabular}

Table 5. Final spaceport approach conditions

\begin{tabular}{ccccccc}
\hline & \multicolumn{3}{c}{ Prestwick } & \multicolumn{3}{c}{ Faroe } \\
Wing area: & $0.6 S_{\text {wing }}$ & $S_{\text {wing }}$ & $1.2 S_{\text {wing }}$ & $0.6 S_{\text {wing }}$ & $S_{\text {wing }}$ & $1.2 S_{\text {wing }}$ \\
\hline Altitude $h(\mathrm{~m})$ & 928 & 442 & 503 & 261 & 648 & 861 \\
Velocity $v(\mathrm{~m} / \mathrm{s})$ & 265 & 294 & 328 & 303 & 297 & 405 \\
Mach & 0.788 & 0.87 & 0.97 & 0.89 & 0.88 & 1.20 \\
Flight path angle $\gamma(\mathrm{deg})$ & -14.00 & -19.95 & -18.19 & -17.79 & -19.04 & -19.71 \\
Downrange distance $(\mathrm{km})$ & 1332 & $\mathbf{1 3 4 3}$ & 961 & 1079 & 1167 & 716 \\
\hline
\end{tabular}

\section{Acknowledgements}

This work was funded by the UK Space Agency through the National Space Technology Programme (NSTP-2) Sub-Orbital and Small Launcher Research Projects Call. Input was also provided by consortium members Reaction Engines Ltd, Clyde Space, Surrey Satellite Technology Ltd and STFC RAL Space into the market demand forecasting, system requirements and design reviews.

\section{References}

\footnotetext{
${ }^{1}$ Niederstrasser, C. and Frick, W., "Small Launch Vehicles- A 2015 State of the Industry Survey," AIAA Small Satellite Conference, 2015.

${ }^{2}$ Toso, F. and Maddock, C., "Deployed payload analysis for a single stage to orbit spaceplane," International Astronautical Conference, 2016.
} 


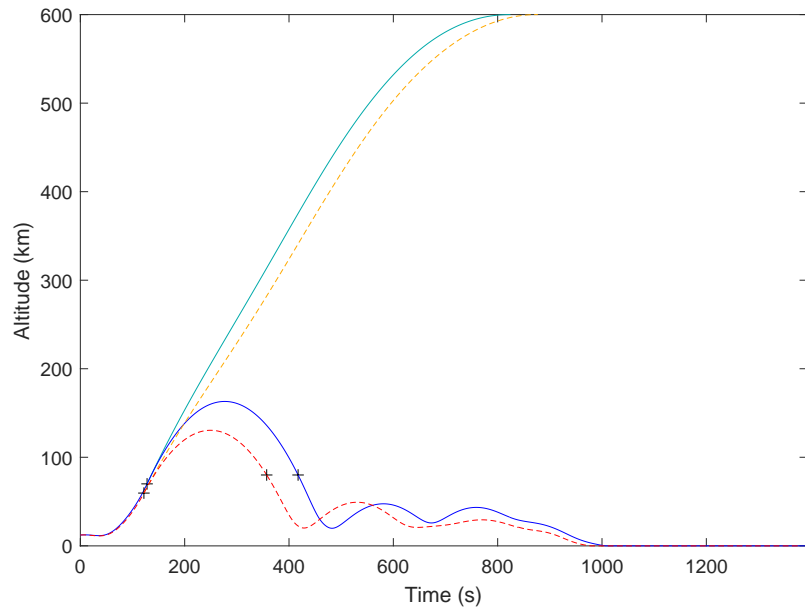

(a) Altitude

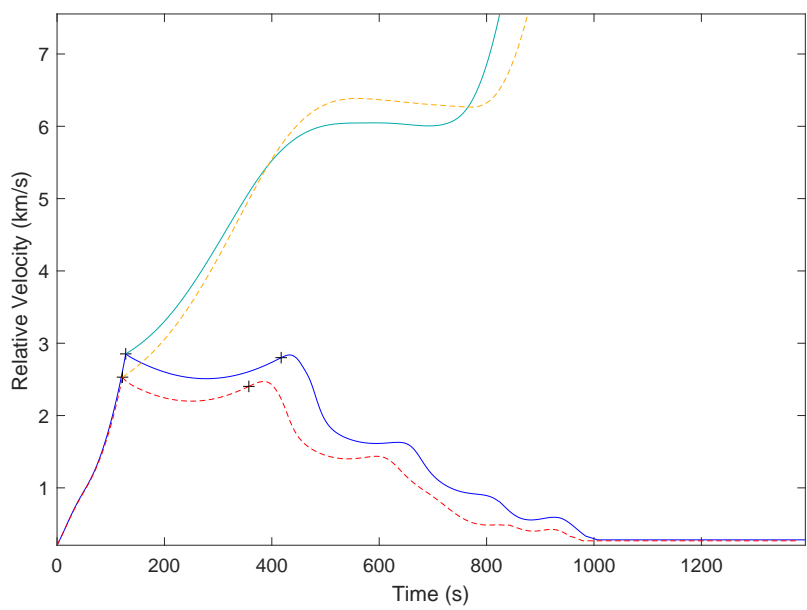

(c) Velocity

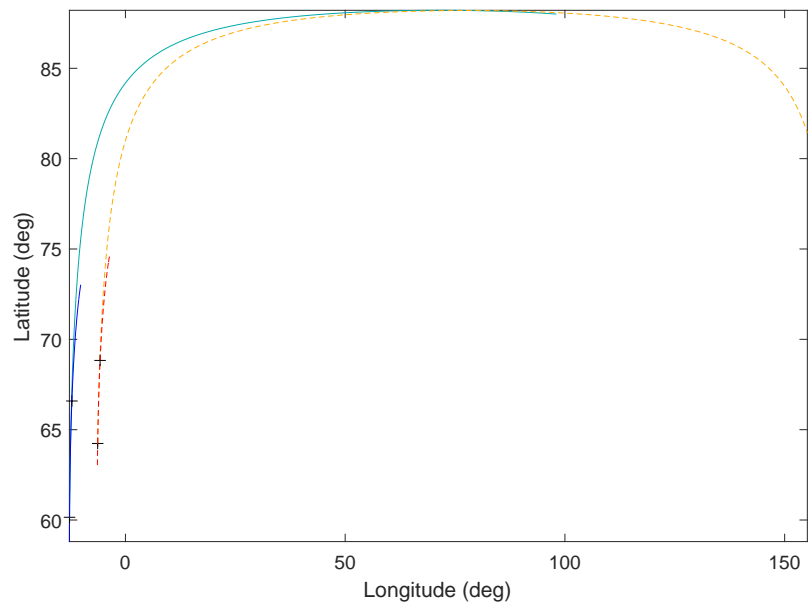

(e) Latitude vs longitude

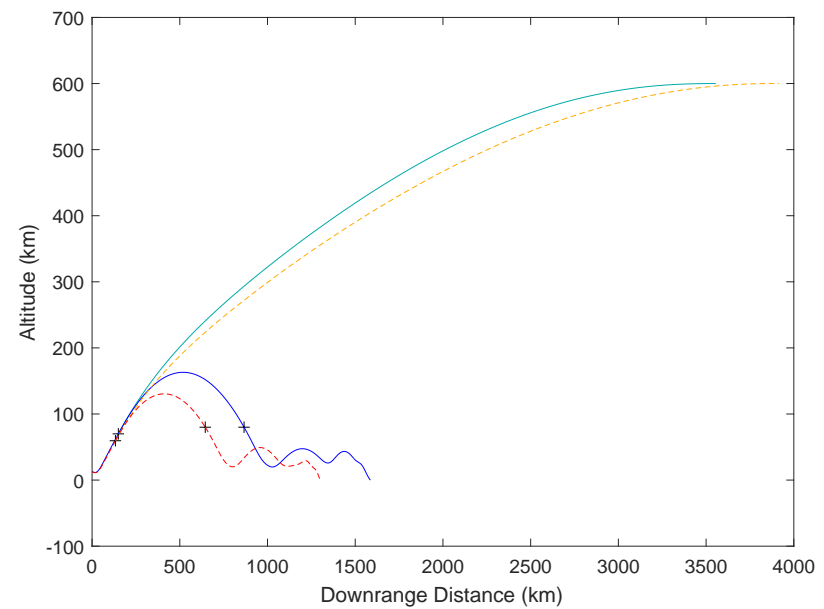

(b) Altitude vs groundtrack

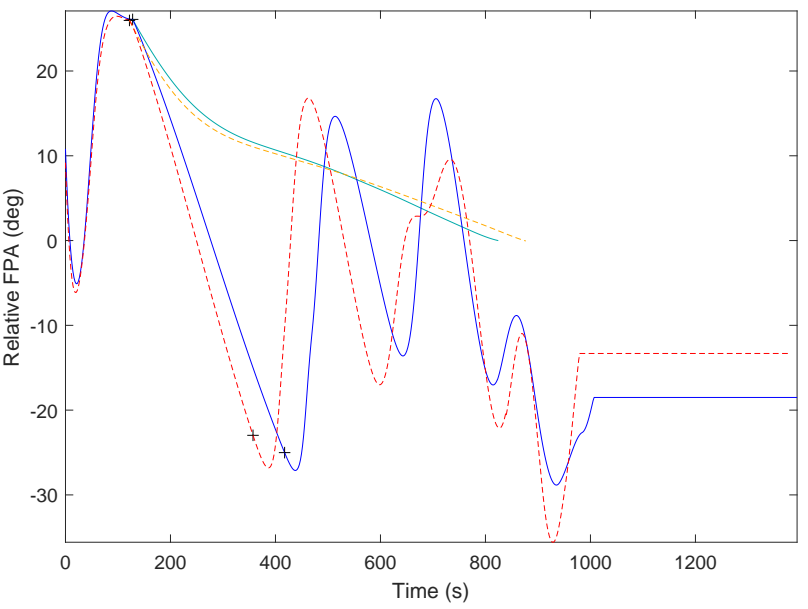

(d) Flight path angle

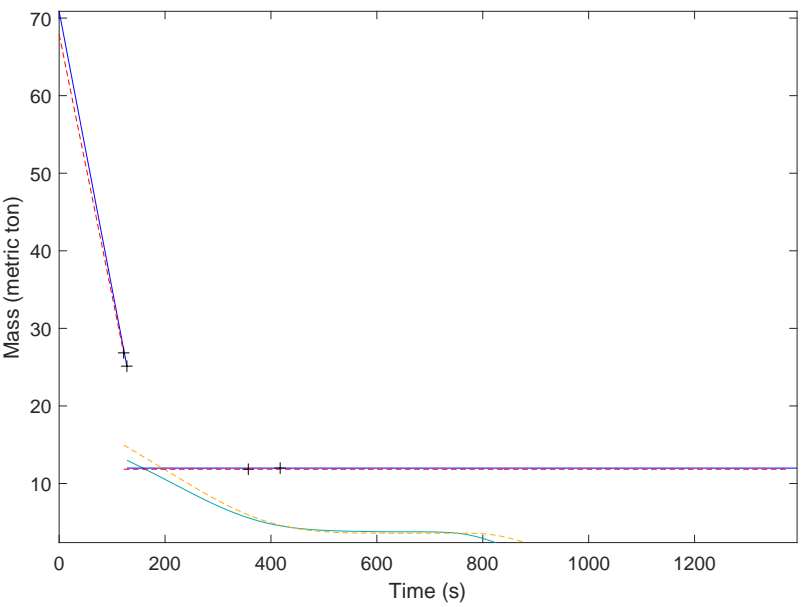

(f) Vehicle mass

Figure 8. Trajectory results for reference case, $1.0 S_{\text {wing }}$ departing from Prestwick (blue/green solid lines) and Faroe (red/orange dotted lines). Start/end of phases are indicated by crosses. 


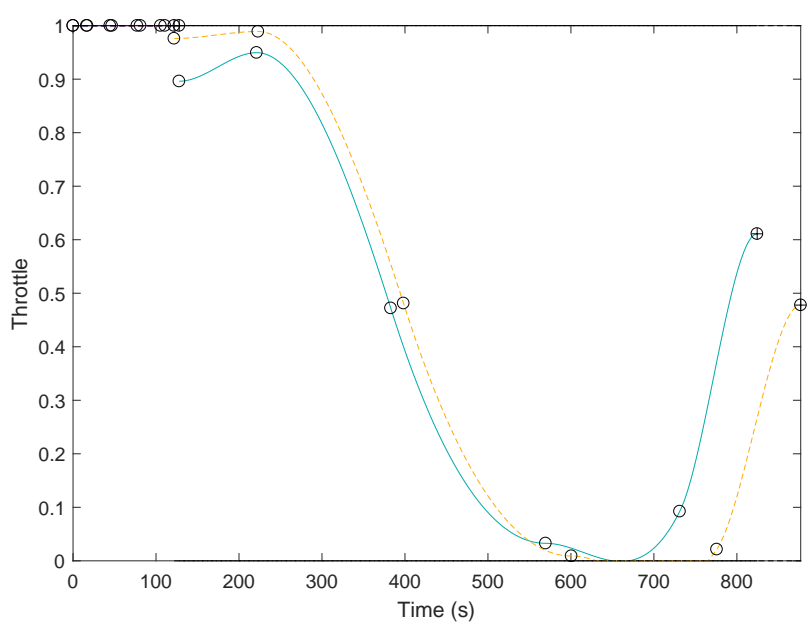

(a) Throttle

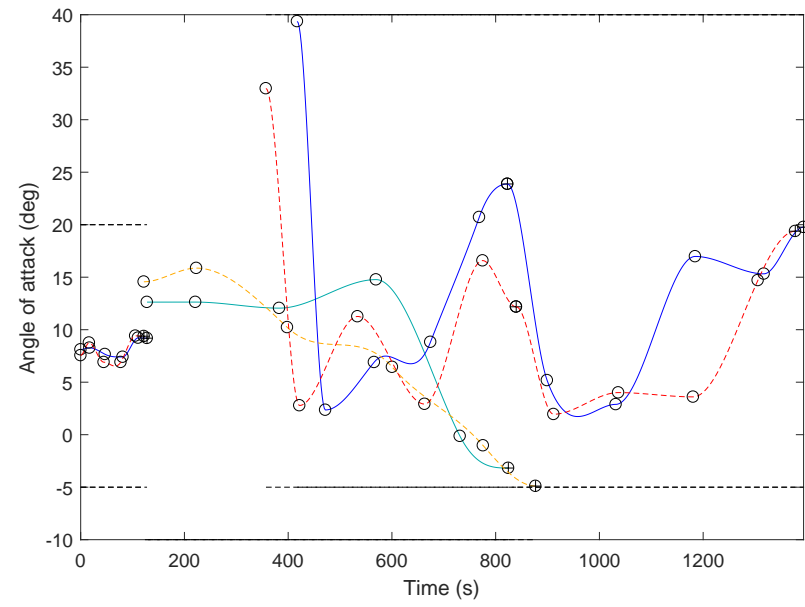

(c) Angle of attack

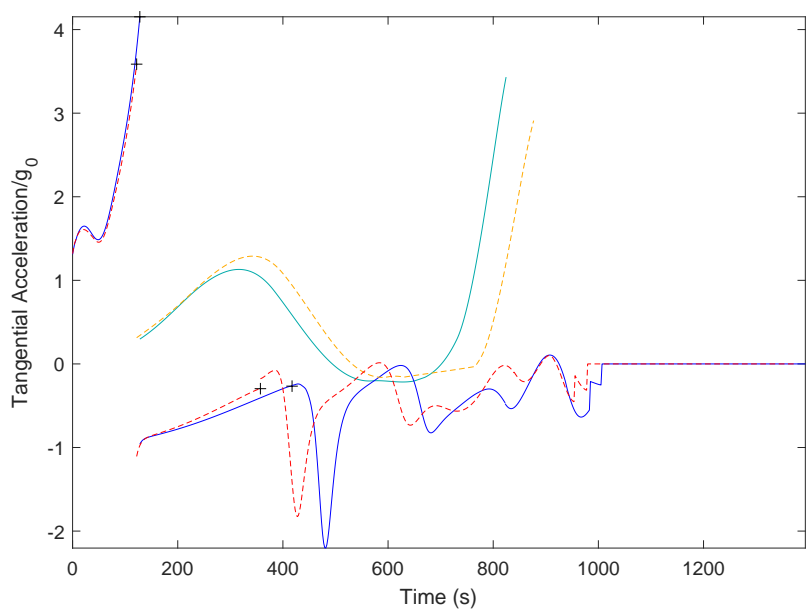

(e) Axial acceleration

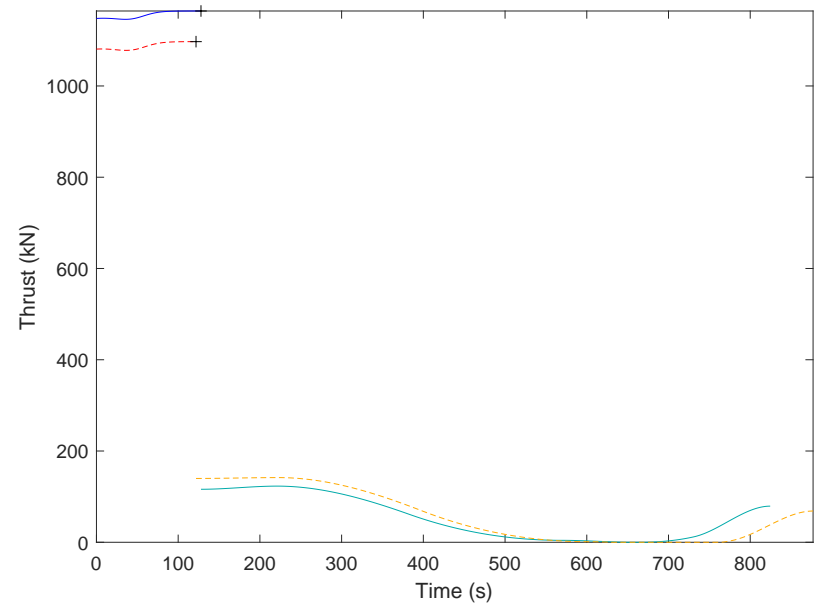

(b) Applied thrust

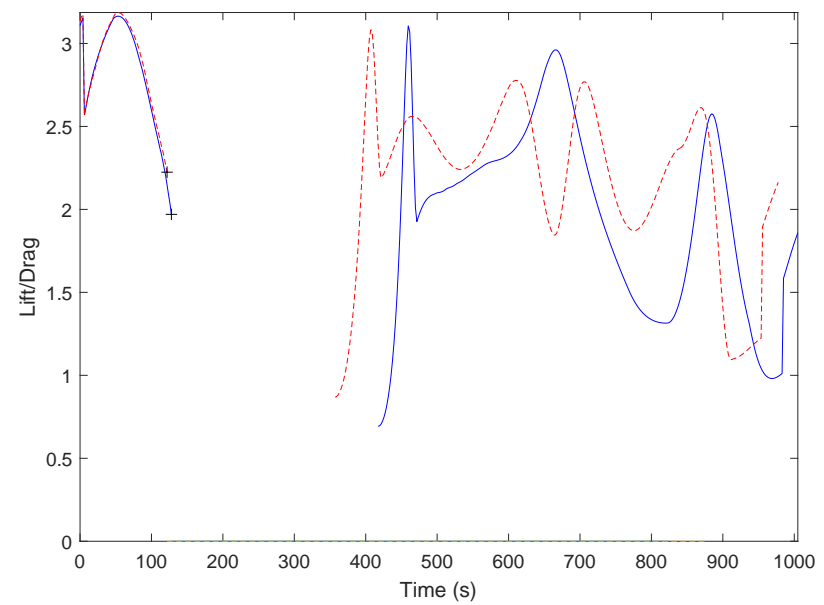

(d) Lift-to-drag ratio

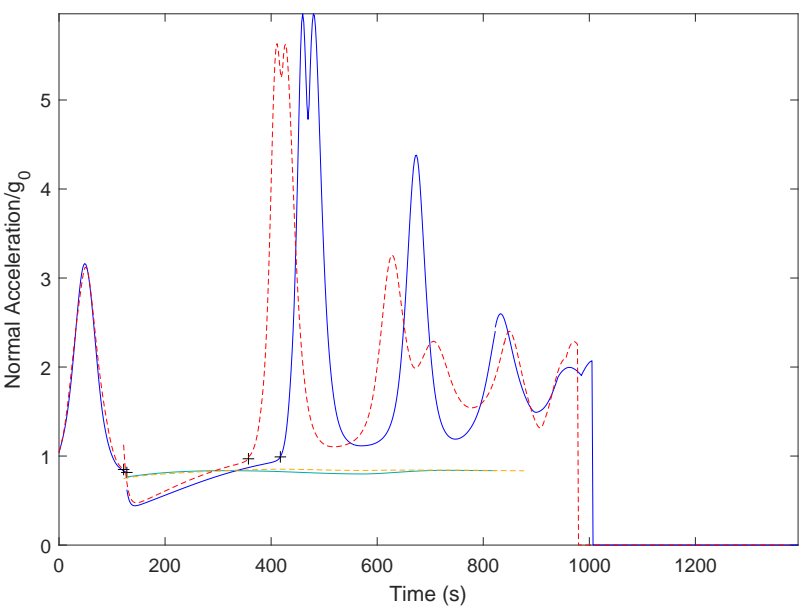

(f) Normal acceleration

Figure 9. Control laws, forces and accelerations for reference case, $1.0 S_{\text {wing }}$ departing from Prestwick (blue/green solid lines) and Faroe (red/orange dotted lines). Start/end of phases are indicated by crosses. 


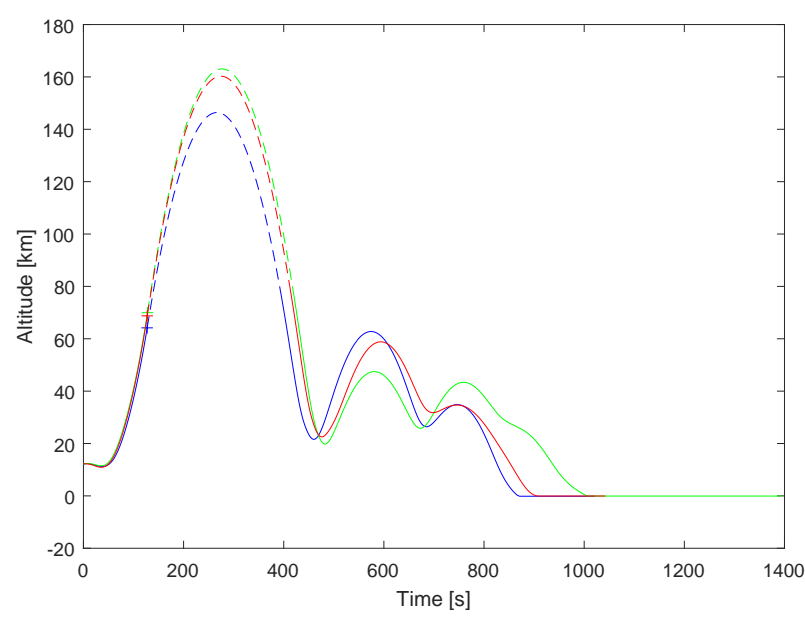

(a) Altitude over time

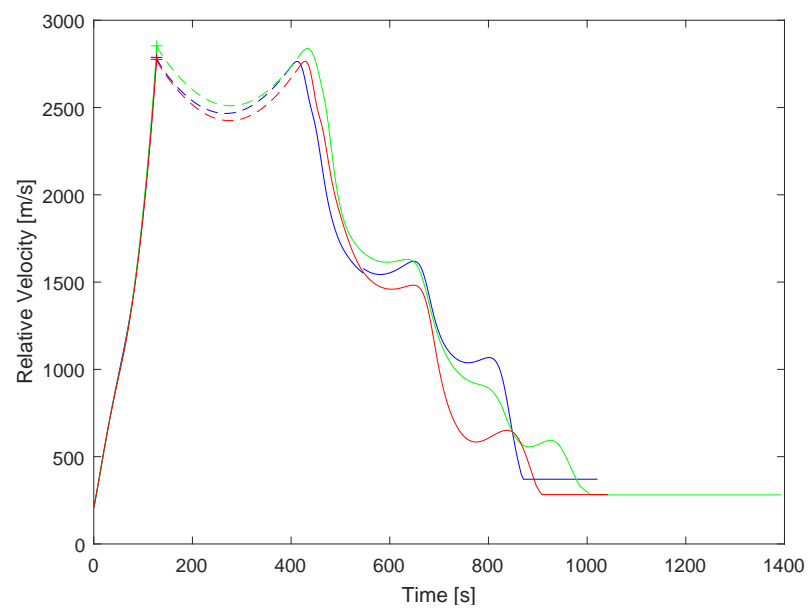

(c) Velocity

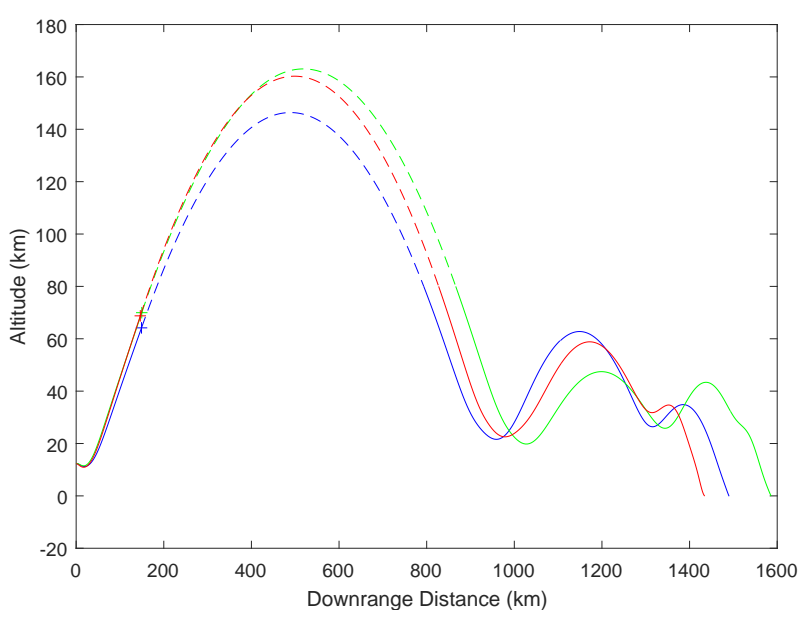

(b) Altitude against groundtrack

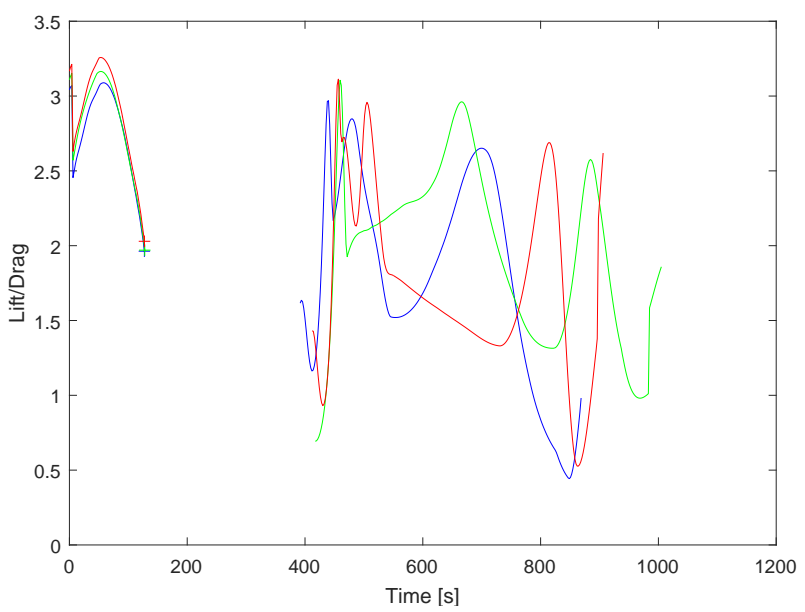

(d) Lift-to-drag ratio

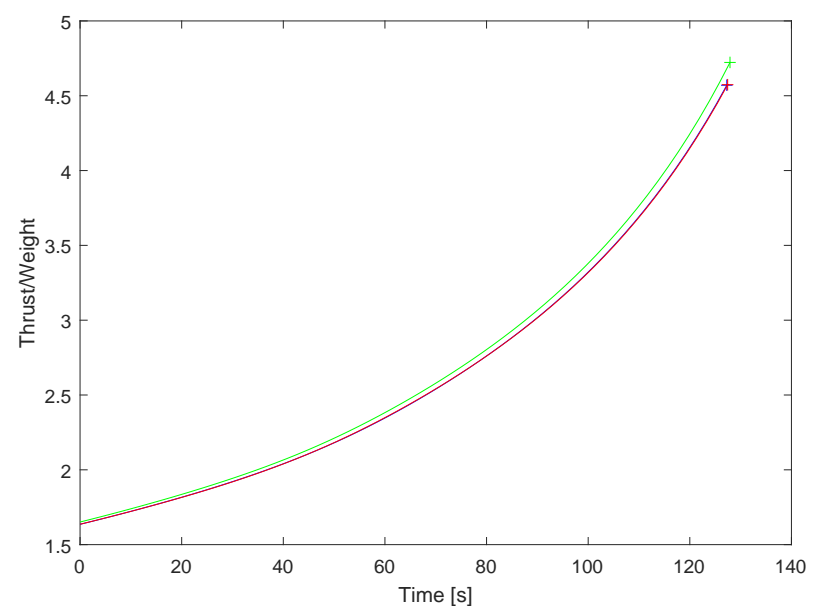

(e) Thrust-to-weight ratio

Figure 10. Comparison of trajectory and design parameters for different wing surface areas: blue $0.6 S_{w i n g}$ (blue), $1.0 S_{\text {wing }}$ (green), and $1.2 S_{\text {wing }}$ (red). All trajectories leave from Prestwick, dashed lines indicate ballistic spaceflight segments. 
${ }^{3}$ Ricciardi, L., Toso, F., Vasile, M., and Maddock, C., "Multi-objective optimal control of the ascent trajectories of launch vehicles," Astrodynamics Specialist Conference, AIAA Space and Astronautics Forum and Exposition, 2016.

${ }^{4}$ Toso, F., Maddock, C., and Minisci, E., "Optimisation of Ascent Trajectories for Lifting Body Space Access Vehicles," Space transportation solutions and innovations symposium, International Astronautical Congress, 2015.

${ }^{5}$ Garner, R., Toso, F., and Maddock, C., "Comparison of the emissions of current expendable launch vehicles and future spaceplanes," International Astronautical Conference, 2016.

${ }^{6}$ Maddock, C., Kontis, K., McIntyre, S., West, M., Feast, S., and Evans, D., "How to launch small payloads? Evaluation of current and future small payload launch systems," Reinventing Space Conference, 2016.

${ }^{7}$ Maddock, C., West, M., Kontis, K., Feast, S., Evans, D., and McIntyre, S., "A commercially driven design approach to UK future small payload launch systems," Reinventing Space Conference, 2016.

${ }^{8}$ Rohrschneider, R., "Development of a mass estimating relationship database for launch vehicle conceptual design," AE8900 Special Project, School of Aerospace Engineering, Georgia Institute of Technology, 2002.

${ }^{9}$ MacConochie, I. O. and Lepsch Jr, R. A., "Characterization of Subsystems for a WB-003 Single Stage Shuttle," 2002.

${ }^{10}$ Mason, L. A., Devan, L., Moore, F. G., and McMillan, D., "Aerodynamic Design Manual for Tactical Weapons," Tech. rep., DTIC Document, 1981.

${ }^{11}$ Fleeman, E., Tactical missile design, AIAA Education Series, 2001.

${ }^{12}$ Fay, J. A. and Riddel, F. R., "Theory of stagnation point heat transfer in dissociated air," Journal of the Aerospace Sciences, Vol. 25, No. 2, 1958, pp. 73-85.

${ }^{13}$ Billig, F. S., "Shock wave shapes around spherical and cylindrical nosed bodies," Journal of Spacecraft and Rockets, Vol. 4, No. 6, 1967, pp. 822-823.

${ }^{14}$ Jorgensen, L. H., "A method for estimating static aerodynamic characteristics for slender bodies of circular and noncircular cross section alone and with lifting surfaces at angles of attack from $0^{\circ}$ TO 90," Tech. Rep. NASA TN D- $7228,1973$.

${ }^{15}$ Singh, A., Experimental study of slender vehicles at hypersonic speeds, Ph.D. thesis, Cranfield University, 1996.

${ }^{16}$ Detra, R., "Generalized Heat Transfer Formulas and Graphs for Nose Cone Re-Entry Into the Atmosphere," ARS Journal, Vol. 31, No. 3, 1961, pp. 318-321.

${ }^{17} \mathrm{SAE}$ Committee AC-9, A. E. S., SAE aerospace applied thermodynamics manual, Society of automotive engineers, 1969.

${ }^{18}$ Schaaf, S. A., Developments in heat transfer, chap. 7, The MIT Press, 1964.

${ }^{19}$ Gordon, S. and McBride, B., "Computer Program for Calculation of Complex Chemical Equilibrium Compositions and Applications. Part 2. Users Manual and Program Description," NASA Office of Management, Scientific and Technical Information Program, Vol. NASA PR 1311 pt2, 1996.

${ }^{20}$ Tewari, A., Atmospheric and space flight dynamics, Springer, 2007.

${ }^{21}$ AIAA Guide to Reference and Standard Atmosphere Models (G-003C-2010e), American Institute of Aeronautics and Astronautics, 2013.

${ }^{22}$ Vinh, N., Optimal trajectories in atmospheric flight, Vol. 2, Elsevier, 2012. 